\title{
A Mass, Momentum, and Energy Conserving, Fully Implicit, Scalable Algorithm for the Multi-Dimensional, Multi-Species Rosenbluth-Fokker-Planck Equation
}

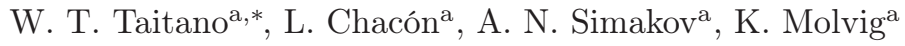 \\ ${ }^{a}$ Los Alamos National Laboratory, Los Alamos, NM 87545
}

\begin{abstract}
In this study, we demonstrate a fully implicit algorithm for the multi-species, multidimensional RosenbluthFokker-Planck equation which is exactly mass-, momentum-, and energy-conserving, and which preserves positivity. Unlike most earlier studies, we base our development on the Rosenbluth (rather than Landau) form of the Fokker-Planck collision operator, which reduces complexity while allowing for an optimal fully implicit treatment. Our discrete conservation strategy employs nonlinear constraints that force the continuum symmetries of the collision operator to be satisfied upon discretization. We converge the resulting nonlinear system iteratively using Jacobian-free Newton-Krylov methods, effectively preconditioned with multigrid methods for efficiency. Single- and multi-species numerical examples demonstrate the advertised accuracy properties of the scheme, and the superior algorithmic performance of our approach. In particular, the discretization approach is numerically shown to be second-order accurate in time and velocity space and and to exhibit manifestly postive entropy production. That is, H-theorem behavior is indicated for all the examples we have tested. The solution approach is demonstrated to scale optimally with respect to grid refinement (with CPU time growing linearly with the number of mesh points), and timestep (showing very weak dependence of CPU time with time-step size). As a result, the proposed algorithm delivers several orders-of-magnitude speedup vs. explicit algorithms.
\end{abstract}

Keywords: Conservative discretization, Fokker-Planck, Rosenbluth potentials, fully implicit algorithms, Jacobian-free Newton-Krylov, multigrid preconditioning

PACS:

\section{Introduction}

The Rosenbluth-Fokker-Planck (or, equivalently, the Fokker-Planck-Landau) collision operator describes the local collisional relaxation process of particle distribution functions (PDFs) in plasmas under the assumption of binary, grazing-angle collisions $[1,2,3,4,5,6]$. This operator, when evolved together with Vlasov's equation and coupled to Maxwell's equations, is regarded as a first-principles physical model for weakly coupled plasmas in all collisionality regimes, and accordingly has a wide range of applications in laboratory (e.g., magnetic and inertial thermonuclear fusion), space (e.g., Earth's magnetosphere), and astrophysical (e.g., solar coronal mass ejections) plasmas.

Mathematically, the Fokker-Planck collision operator is physically well posed in several ways. It enforces strict conservation of mass, momentum, and energy; it preserves positivity of the PDF (which lives in the position-velocity phase space); and it satisfies the Boltzmann H-theorem [7], which forces entropy in the system to grow monotonically except at collisional equilibrium. Thus, the well-known Maxwellian distribution function is the equilibrium solution of the Fokker-Planck collision operator.

However, despite its favorable mathematical properties, the Fokker-Planck collision operator is a nonlinear, integro-differential, stiff advection-diffusion operator in velocity space, which is remarkably difficult to

* Corresponding author

Email address: taitano@lanl.gov (W. T. Taitano) 
deal with numerically. The numerical difficulties posed by the Fokker-Planck collision operator manifest in two fundamental ways: 1) the enforcement of its sound mathematical properties in the discrete, and 2) its numerical stiffness, which challenges the development of efficient temporal integration schemes. Both have received ample attention in the literature.

The nonlinear, integro-differential nature of the Fokker-Planck collision operator stems from the fact that both diffusion and advection coefficients are defined as integrals of the PDF. The Fokker-Planck-Landau (FPL) form [6] considers a direct integral formulation for these coefficients. The Rosenbluth-Fokker-Planck (RFP) form [1], on the other hand, casts the integral relationships as Poisson equations. Both are otherwise equivalent in the continuum, but they feature very different properties and challenges in the discrete. In particular, the symmetries present in FPL make it ideally suited for the development of conserving numerical schemes, while the Poisson form in RFP make it more suitable for fast-solver development.

The preservation of conservation laws, positivity, and the H-theorem in the numerical discretization of the Fokker-Planck collision operator has been the subject of many studies for decades. Chang and Cooper [8] developed a positivity-preserving scheme for the linearized 1D FPL equation, later made energy-preserving by Langdon [9] , and tested numerically by Kho [10]. Larsen et al. [11] and Epperlein [12] later extended the Chang and Cooper approach to the nonlinear case, for which Chang-Cooper could develop negative solutions, by pursuing a fully implicit approach. The extension of Chang-Cooper's positivity-preserving scheme to the 2D FPL equation in cylindrical geometry has been attempted recently by Yoon and Chang [13, 14]. While complex, the method succeeds in preserving the analytical Maxwellian equilibrium solution, but, as ChangCooper, it Picard-linearizes the FLE equation and cannot guarantee positivity when the solution is far away from equilibrium. Regarding the H-theorem, one important line of study is that of "entropic" schemes for the Fokker-Planck equation. These are schemes that guarantee an increasing entropy, and are all based on the FPL form. The analytical form of the FP equation used as a starting point for these schemes was first proposed by Pekker [15]. Specific numerical schemes have been proposed by various authors for the one-dimensional (1D) $[16,17,18]$ and multi-dimensional $[19,20]$ FPL equation. Entropic schemes, however, are all based on the logarithm of the PDF, and are therefore limited when dealing with distribution functions that vanish anywhere in the domain.

In regards to the numerical stiffness of the Fokker-Planck collision operator, many authors have realized the potential efficiency benefits of implicit time discretization approaches. In principle, these are able to timestep over fast numerical time scales while resolving dynamical time scales of interest. Linearly implicit methods are common in the literature [16, 19, 21, 22, 23, 24, 25]. These split the integro-differential system into an integral step (to find coefficients) and a differential one (to advance the advection-diffusion equation). However, accuracy considerations limit the practical time step that can be employed, as such formulations usually fail to obtain the correct equilibrium solution in the limit of $\Delta t \nu_{\text {coll }} \gg 1$ (with $\Delta t$ the time step, and $\nu_{\text {coll }}$ the characteristic collision frequency), i.e., in the limit of strong collisionality or large enough implicit time steps.

The desired ability of implicit methods to take large time steps puts a premium on strict conservation and nonlinear convergence, as in this way the numerical method is guaranteed to yield the correct asymptotic solution. The advantages of fully implicit schemes, combined with strict energy conservation, have been demonstrated in various 1D Fokker-Planck studies [11, 12]. However, fully implicit schemes applied to the multi-D Fokker-Planck-Landau equation present a serious challenge. Owing to the integro-differential nature of the FPL equation, implicit schemes result in a large, nonlinearly coupled set of algebraic equations that produce dense matrices, and lead to prohibitive scalings of the computational complexity $\left(\mathcal{O}\left(N_{v}^{2}\right)\right.$, with $N_{v}$ the total number of velocity-space mesh points). Fast schemes [of $\mathcal{O}\left(N_{v} \log N_{v}\right)$ ] have been developed for the evaluation of the Fokker-Planck-Landau collision operator, based either on spectral representations [26] or an adaption of multigrid methods [19]. Spectral methods that have been developed for the FPL equation to date are explicit in time, however, and when accounting for explicit time-step-stability constraints, they result in an $\mathcal{O}\left(N_{v}^{1+2 / d}\right)$ scaling of the computational complexity, with $d$ the dimensionality in velocity space. Multigrid implementations for the FPL equation are typically linearly implicit (and thus timestep-limited for accuracy), and coefficient integrals are computed either by Monte Carlo [19] (which introduces a random component) or fast-multipole methods [21] (which, while scalable, can become expensive).

There is a school of thought in the context of asymptotic-preserving (AP) methods that seeks to accelerate the solution of the FPL equation by so-called penalization techniques [27, 28] . These methods apply a penalization operator that captures the stiffest components of the collision operator, but is linear and much 
simpler to invert. In the context of the FPL equation, the collision operator is a modified diffusion operator in velocity space [28]. In strongly collisional regimes, AP methods (as defined in the references) guarantee that the penalized FPL solution is a Maxwellian, $f_{M}$, to $\mathcal{O}(\epsilon)$ [27], with $\epsilon$ a measure of the collisional time scale normalized to the characteristic time scale of interest $(\epsilon \ll 1$ in strongly collisional regimes). However, accurate transport computations in collisional regimes require computing $f$ to $\mathcal{O}\left(\epsilon^{2}\right)[29,30,31]$ and to our knowledge, there are no variants of AP schemes which satisfy this.

Unlike the Landau form, the Rosenbluth-Fokker-Planck formulation of the collision operator naturally leads to a scalable, fast implementation, with computational complexity of $\mathcal{O}\left(N_{v}\right)$, provided that a fast Poisson solver in an infinite velocity space is available. Several strategies for this have been put forth in the literature, including classical multigrid methods (author?) [32] (with far-field boundary conditions, discussed further in this study), integral approaches [33], or by solving the Rosenbluth potentials in the residual formulation [22]. The challenge with the RFP approach is to embed the conservation properties in the numerical scheme, as symmetries of the collision operator that lead to these properties are obscured by the potential formulation. In fact, to our knowledge, so far there has only been an attempt to make the multidimensional RFP formulation energy-conserving, with mixed results due to the impact of boundary conditions [34].

In this study, we address both the discrete conservation and the temporal stiffness challenges for the multidimensional Rosenbluth-Fokker-Planck formulation. In particular, we propose a fully implicit, nonlinearly converged algorithm for the multi-dimensional, multi-species Rosenbluth-Fokker-Planck equation that ensures exact mass, momentum, and energy conservation, preserves positivity, and satisfies the H-theorem numerically. The discretization is based on flux-limited, conservative finite differences, and exploits the advection-diffusion nature of the collision operator to ensure positivity for arbitrary implicit time steps. Our approach improves on that of Ref. [34] in that we directly solve the diffusion-advection form of the Fokker-Planck operator (rather than transforming the friction term into a nonlinear tensor operator), and we achieve exact conservation properties. These conservation properties are guaranteed by discretely enforcing the underlying symmetries of the collision operator (which are evident in the FPL form, but not in the RFP one). Strict conservation properties ensure that the correct numerical equilibrium is achieved. Numerical experiments demonstrate that the entropy grows monotonically except at collisional equilibrium. The proposed discrete formulation of the Fokker-Planck collision operator does not require an implicit temporal update to ensure preservation of these properties (in fact, these properties survive with explicit time stepping). Nevertheless, the combination of exact conservation properties and an efficient nonlinear implicit solver delivers an algorithm which is optimal $\left[\mathcal{O}\left(N_{v}\right)\right]$, second-order accurate in time and velocity space, and asymptotic-preserving to $\mathcal{O}\left(\epsilon^{2}\right)$. Our nonlinear solver strategy follows Ref. [32] , and is based on Jacobian-free Newton-Krylov (JFNK) methods, preconditioned with classical multigrid (MG) methods [35] for efficiency. Key to our implementation is the nonlinear enslavement of the Rosenbluth-potentials Poisson solves, which together with the Picard linearization of the diffusion and advection coefficients, effectively decouples all species and renders a simple block-diagonal preconditioning operator, of straightforward inversion with MG. The resulting preconditioner is shown to deliver mesh-independent convergence rates, and features a very weak dependence with time-step size.

The rest of the paper is organized as follows. Section 2 introduces the RFP equation and its main properties. The conservative, positivity-preserving discretization of the RFP collision operator is discussed in Sec. 3. The implicit discretization and the solver strategy are discussed in Sec. 4. The numerical performance of the scheme, both in accuracy and efficiency, is demonstrated with various single- and multispecies tests in Sec. 5. Finally, we conclude in Sec. 6.

\section{The multi-species Rosenbluth-Fokker-Planck equation}

Collisional relaxation in a multi-species plasma is described by the Fokker-Planck equation, which for plasma species $\alpha$ described by the particle distribution function, $f_{\alpha}(\vec{v}, t)$, in velocity space, $\vec{v}$, reads:

$$
\frac{\partial f_{\alpha}}{\partial t}=\sum_{\beta}^{N_{s}} C_{\alpha \beta},
$$


where $N_{s}$ is the total number of plasma species in the system, and $C_{\alpha \beta}$ is the Fokker-Planck collision operator for species $\alpha$ colliding with species $\beta$ :

$$
C_{\alpha \beta}=\Gamma_{\alpha \beta} \vec{\nabla}_{v} \cdot\left[\overleftrightarrow{D}_{\beta} \cdot \nabla_{v} f_{\alpha}-\frac{m_{\alpha}}{m_{\beta}} \vec{A}_{\beta} f_{\alpha}\right]
$$

Here, $\Gamma_{\alpha \beta}=\frac{2 \pi Z_{\alpha}^{2} Z_{\beta}^{2} e^{4} \Lambda_{\alpha \beta}}{m_{\alpha}^{2}}, \stackrel{\leftrightarrow}{D} \beta$ and $\vec{A}_{\beta}$ are the tensor-diffusion and friction coefficients for species $\beta, m_{\alpha}$ and $m_{\beta}$ are the masses of species $\alpha$ and $\beta, Z_{\alpha}$ and $Z_{\beta}$ are the ionization state of species $\alpha$ and $\beta, e$ is the proton charge, and $\Lambda_{\alpha \beta}$ is the Coulomb logarithm $\left(\Lambda_{\alpha \beta}=\Lambda_{\beta \alpha}=10\right.$ is assumed in this study). In this manuscript, we normalize all quantities with respect to a reference charge $e_{0}$, a reference mass $m_{0}$, and a reference temperature $T_{0}$.

In the Rosenbluth-Fokker-Planck formulation, the velocity-space-transport coefficients are computed from the Rosenbluth potentials $G_{\beta}, H_{\beta}$ as:

$$
\begin{aligned}
\overleftrightarrow{D}_{\beta} & =\nabla_{v} \nabla_{v} G_{\beta}, \\
\vec{A}_{\beta} & =\nabla_{v} H_{\beta},
\end{aligned}
$$

and the Rosenbluth potentials are in turn computed from the distribution function of species $\beta$ as:

$$
\begin{gathered}
\nabla_{v}^{2} H_{\beta}=-8 \pi f_{\beta}, \\
\nabla_{v}^{2} G_{\beta}=H_{\beta} .
\end{gathered}
$$

The Fokker-Planck collision operator preserves the positivity of $f$, and conserves mass, momentum, and energy. These conservation properties stem from the following symmetries [30]:

$$
\begin{aligned}
\left\langle 1, C_{\alpha \beta}\right\rangle_{\vec{v}} & =0, \\
m_{\alpha}\left\langle\vec{v}, C_{\alpha \beta}\right\rangle_{\vec{v}} & =-m_{\beta}\left\langle\vec{v}, C_{\beta \alpha}\right\rangle_{\vec{v}}, \\
m_{\alpha}\left\langle\frac{v^{2}}{2}, C_{\alpha \beta}\right\rangle_{\vec{v}} & =-m_{\beta}\left\langle\frac{v^{2}}{2}, C_{\beta \alpha}\right\rangle_{\vec{v}}
\end{aligned}
$$

where the inner product is defined as $\langle A, B\rangle_{\vec{v}}=2 \pi \int_{-\infty}^{\infty} d v_{\|} \int_{0}^{\infty} d v_{\perp} v_{\perp} A(\vec{v}) B(\vec{v})$ for a cylindrically symmetric domain. The collision operator also satisfies the so-called $\mathrm{H}$-theorem, which guarantees that the total entropy of the system, $S=-\sum_{\alpha}\left\langle\ln \left|f_{\alpha}\right|, f_{\alpha}\right\rangle_{\vec{v}}$, increases monotonically with time, unless all $f_{\alpha}$ are Maxwellian with a common temperature.

Enforcing these symmetries in the discrete will be essential to guarantee these conservation properties numerically. We discuss the numerical discretization of the collision operator next.

\section{Conservative discretization of the Fokker-Planck collision operator}

In this manuscript, we consider a finite-volume approach to discretize the Fokker-Planck collision operator. For discretization purposes, and with a future extension to $1 \mathrm{D}-2 \mathrm{~V}$ in mind, we consider a $2 \mathrm{~V}$ cylindrical velocity space $\left(v_{\|}\right.$and $\left.v_{\perp}\right)$ with azimuthal symmetry (Fig. 3.1). Here, $v_{\|}$is the coordinate along the cylindrical axis, while $v_{\perp}$ acts as the radial coordinate. The discrete volume for cell $(j, k)$ is computed as:

$$
\Delta V_{j, k}=2 \pi v_{\perp, k} \Delta v_{\|, j} \Delta v_{\perp, k},
$$

where $\Delta v_{\|, j}$ and $\Delta v_{\perp, k}$ are the discrete velocity space cell sizes in the parallel and perpendicular directions, respectively. For a uniform mesh, we have:

$$
\Delta v_{\|}=\frac{L_{\|}}{N_{\|}}, \Delta v_{\perp}=\frac{L_{\perp}}{N_{\perp}}
$$

where $L_{\|}$and $L_{\perp}$ are the parallel and perpendicular velocity space domain sizes, and $N_{\|}$and $N_{\perp}$ are the number of cells in the respective directions. The mesh is arranged such that cell faces map to the domain boundary (and therefore outermost cell centers are half a mesh-spacing away from the boundary). The 


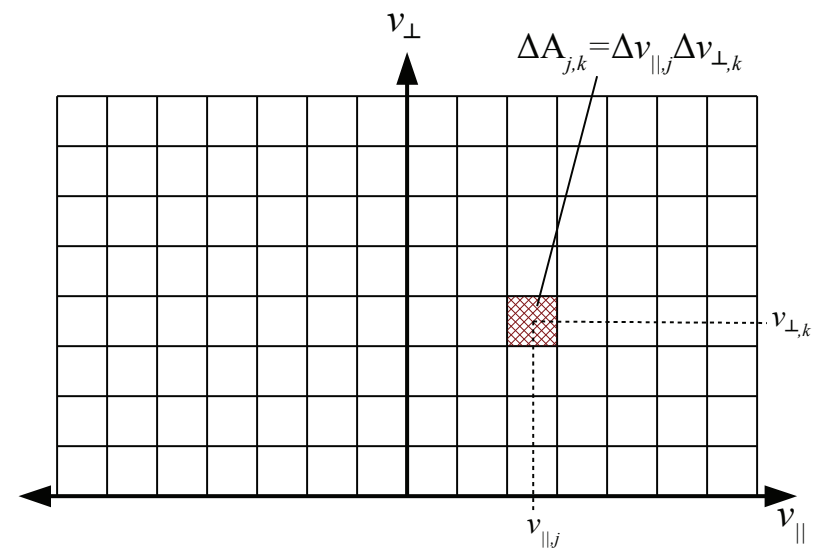

Figure 3.1: 0D2V cylindrical velocity space grid with azimuthal symmetry.

distribution function $f$ and the Rosenbluth potentials $H, G$ are defined at cell centers. Velocity-space inner products are approximated via a mid-point quadrature rule as:

$$
\langle A, B\rangle_{\vec{v}} \approx 2 \pi \sum_{j=1}^{N_{\|}} \sum_{k=1}^{N_{\perp}} v_{\perp, k} \Delta v_{\|, j} \Delta v_{\perp, k} A_{j, k} B_{j, k} .
$$

\subsection{Finite-volume discretization of the collision operator}

The Fokker-Planck operator can be written as:

$$
C_{\alpha \beta}=\vec{\nabla}_{v} \cdot \vec{J}_{\alpha \beta}
$$

where:

$$
\vec{J}_{\alpha \beta}=\Gamma_{\alpha \beta}\left[\vec{J}_{G, \alpha \beta}-\frac{m_{\alpha}}{m_{\beta}} \vec{J}_{H, \alpha \beta}\right]
$$

is the Fokker-Planck flux, and:

$$
\begin{aligned}
\vec{J}_{G, \alpha \beta} & =\overleftrightarrow{D}_{\beta} \cdot \nabla_{v} f_{\alpha} \\
\vec{J}_{H, \alpha \beta} & =\vec{A}_{\beta} f_{\alpha}
\end{aligned}
$$

are the collisional inter-species diffusion and friction fluxes, respectively. We discretize Eq. 3.2 in flux-form in a cylindrical velocity space as (Fig. 3.2):

$$
C_{\alpha \beta, j, k}=\frac{J_{\|, \alpha \beta, j+1 / 2, k}-J_{\|, \alpha \beta, j-1 / 2, k}}{\Delta v_{\|, j}}+\frac{v_{\perp, k+1 / 2} J_{\perp, \alpha \beta, j, k+1 / 2}-v_{\perp, k-1 / 2} J_{\perp, \alpha \beta, j, k-1 / 2}}{v_{\perp, k} \Delta v_{\perp, k}} .
$$

Fluxes at cell faces are given by:

$$
\begin{aligned}
J_{\|, G, \alpha \beta, j+\frac{1}{2}, k} & =\left(D_{\|\|, \beta} \partial_{v_{\|}} f_{\alpha}+D_{\| \perp, \beta} \partial_{v_{\perp}} f_{\alpha}\right)_{j+\frac{1}{2}, k}, \\
J_{\perp, G, \alpha \beta, j, k+\frac{1}{2}} & =\left(D_{\perp \|, \beta} \partial_{v_{\|}} f_{\alpha}+D_{\perp \perp, \beta} \partial_{v_{\perp}} f_{\alpha}\right)_{j, k+\frac{1}{2}}, \\
J_{\|, H, \alpha \beta, j+\frac{1}{2}, k} & =A_{\|, \beta, j+\frac{1}{2}, k} f_{\alpha, j+\frac{1}{2}, k}, \\
J_{\perp, H, \alpha \beta, j, k+\frac{1}{2}} & =A_{\perp, \beta, j, k+\frac{1}{2}} f_{\alpha, j, k+\frac{1}{2}} .
\end{aligned}
$$

The appropriate discrete form of these fluxes is the key to positivity preservation and strict conservation properties, and is discussed next. 


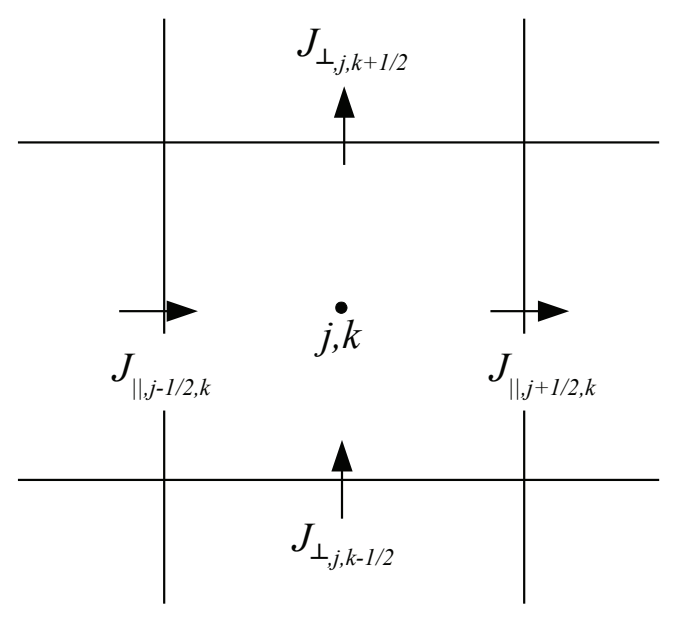

Figure 3.2: Illustration of flux on $2 \mathrm{~V}$ velocity space cell faces in terms of parallel $(\|)$ and perpendicular $(\perp)$ components.

\subsection{Positivity preserving discretization of the Fokker-Planck fluxes}

Earlier attempts at enforcing positivity of the distribution function in $1 \mathrm{D}[8,36]$ and multi-D $[13,14]$ have explored Fokker-Planck flux interpolations compatible with the exact Maxwellian distribution in local thermal equilibrium. While the approach is tractable in 1D, it is intrinsically based on the Landau form of the Fokker-Planck equation (not the Rosenbluth form), and becomes very cumbersome and expensive in multiple dimensions.

The approach pursued here to enforce positivity is to exploit the advection-diffusion structure of the Fokker-Planck collision operator, and tailoring existing discrete positivity preserving schemes for each term. In particular, for the friction operator (which is advective in nature), we have employed the SMART limiter scheme [37] to reconstruct fluxes at cell faces. For the tensor-diffusion operator, we have used a symmetric discretization of the tensor-diffusion fluxes [38], combined with an adaptation of the positivity-preserving limiter proposed in Ref. [39], to reconstruct the off-diagonal terms in $\stackrel{\leftrightarrow}{D} \cdot \nabla_{v} f$ on cell faces. We briefly provide the details of the discretization next.

To construct the friction operator fluxes (Eqs. 3.7, 3.8), we compute the components of $\vec{A}=-\vec{\nabla}_{v} H$ at faces as:

$$
\begin{aligned}
& A_{\|, j+\frac{1}{2}, k}=-\left.\frac{\partial H}{\partial v_{\|}}\right|_{j+1 / 2, k} \approx-\frac{H_{j+1, k}-H_{j, k}}{\Delta v_{\|, j+1 / 2}}, \\
& A_{\perp, j, k+\frac{1}{2}}=-\left.\frac{\partial H}{\partial v_{\perp}}\right|_{j, k+1 / 2} \approx-\frac{H_{j, k+1}-H_{j, k}}{\Delta v_{\perp, k+1 / 2}} .
\end{aligned}
$$

The distribution function values at cell faces, $f_{j+1 / 2, k}$ and $f_{j, k+1 / 2}$ are computed using the SMART limiter (App. AppendixA), with effective velocity $\vec{A}=-\vec{\nabla}_{v} H$.

The diffusion operator fluxes are constructed as proposed in Ref. [38], and summarized in App. AppendixB. Such discretization yields a symmetric, positive-definite matrix, while being numerically well-posed (i.e., does not feature a null space, and diagonal contributions feature a maximum principle). However, off-diagonal contributions do not feature a maximum principle, and therefore the discretization does not guarantee positivity of the solution in general. This is particularly the case in the presence of strong gradients when off-diagonal diffusion coefficients are significant. In order to address this issue, we follow the flux-limiting technique for tensor-diffusion operators proposed in Ref. [39], and adapt it for the symmetric discretization in App. AppendixB (which, unlike the symmetric discretization considered in the reference, does not feature a red-black null space).

The basic idea in Ref. [39] is to perform a nested flux-limiting for the off-diagonal contributions to the 
diffusion flux $D_{\| \perp} \partial_{v_{\perp}} f$ and $D_{\perp \|} \partial_{v_{\|}} f$ in Eqs. 3.5, 3.6 as:

$$
\begin{aligned}
\left(D_{\| \perp} \partial_{v_{\perp}} f\right)_{j+\frac{1}{2}, k}= & M C\left(D_{\| \perp, j+\frac{1}{2}, k+\frac{1}{2}} M C\left[\left.\frac{\partial f}{\partial v_{\perp}}\right|_{j, k+\frac{1}{2}},\left.\frac{\partial f}{\partial v_{\perp}}\right|_{j+1, k+\frac{1}{2}}\right]\right. \\
& \left.D_{\| \perp, j+\frac{1}{2}, k-\frac{1}{2}} M C\left[\left.\frac{\partial f}{\partial v_{\perp}}\right|_{j, k-\frac{1}{2}},\left.\frac{\partial f}{\partial v_{\perp}}\right|_{j+1, k-\frac{1}{2}}\right]\right)
\end{aligned}
$$

and

$$
\begin{aligned}
\left(D_{\perp \|} \partial_{v_{\|}} f\right)_{j, k+\frac{1}{2}}=M C & \left(D_{\perp \|, j+\frac{1}{2}, k+\frac{1}{2}} M C\left[\left.\frac{\partial f}{\partial v_{\|}}\right|_{j+\frac{1}{2}, k},\left.\frac{\partial f}{\partial v_{\|}}\right|_{j+\frac{1}{2}, k+1}\right]\right. \\
& \left.D_{\perp \|, j-\frac{1}{2}, k+\frac{1}{2}} M C\left[\left.\frac{\partial f}{\partial v_{\|}}\right|_{j-\frac{1}{2}, k},\left.\frac{\partial f}{\partial v_{\|}}\right|_{j-\frac{1}{2}, k+1}\right]\right) .
\end{aligned}
$$

Here, $M C$ is the monotonized-central flux limiting scheme [40] which is proposed in Ref. [39] and also considered in this study:

$$
M C(a, b)=\operatorname{minmod}\left[2 \operatorname{minmod}(a, b), \frac{a+b}{2}\right] .
$$

The minmod function is defined as:

$$
\operatorname{minmod}(a, b)= \begin{cases}\min (a, b) & \text { if } \mathrm{a}, \mathrm{b}>0 \\ \max (a, b) & \text { if } \mathrm{a}, \mathrm{b}<0 \\ 0 & \text { if } \mathrm{ab} \leq 0\end{cases}
$$

It can easily be shown that for a smooth solution, the flux-limiting scheme will yield the original symmetric discretization proposed in App. AppendixB.

A related aspect in the preservation of positivity is the temporal discretization employed. The focus of this work is on implicit methods for efficiency. However, except for first-order backward Euler, higher-order implicit methods are not strictly positivity preserving in general [41]. We will assess the impact of the temporal discretization on positivity in the numerical results section (Sec. 5). We will also assess the impact of a finite nonlinear tolerance on positivity.

\subsection{Discrete Mass Conservation}

Mass conservation follows from Eq. 2.7, which gives:

$$
\left\langle 1, C_{\alpha \beta}\right\rangle_{v}=\left\langle 1, \nabla_{v} \cdot \vec{J}_{\alpha \beta}\right\rangle_{v}=\int_{\partial \vec{v}} d S \vec{n} \cdot \vec{J}_{\alpha \beta}=0 .
$$

The condition on the surface integral is enforced by setting the normal component of the flux to zero at the boundary. This is in fact a sufficient set of boundary conditions for the well-posedness of the Fokker-Planck collision operator.

\subsection{Discrete Energy Conservation}

Energy conservation in the Fokker-Planck equation follows from Eq. 2.9:

$$
m_{\alpha}\left\langle\frac{v^{2}}{2}, C_{\alpha \beta}\right\rangle_{\vec{v}}=-m_{\beta}\left\langle\frac{v^{2}}{2}, C_{\beta \alpha}\right\rangle_{\vec{v}} .
$$

Substituting in $C_{\alpha \beta}$ and $C_{\beta \alpha}$, and integrating by parts gives:

$$
m_{\alpha} \Gamma_{\alpha \beta}\left\langle\vec{v}, \vec{J}_{G, \alpha \beta}-\frac{m_{\alpha}}{m_{\beta}} \vec{J}_{H, \alpha \beta}\right\rangle_{\vec{v}}=-m_{\beta} \Gamma_{\beta \alpha}\left\langle\vec{v}, \vec{J}_{G, \beta \alpha}-\frac{m_{\beta}}{m_{\alpha}} \vec{J}_{H, \beta \alpha}\right\rangle_{\vec{v}} .
$$


Substituting the definition of $\Gamma_{\alpha \beta}$ and gathering terms, we obtain:

$$
\frac{1}{m_{\alpha}}\left\langle\vec{v}, \vec{J}_{G, \alpha \beta}-\vec{J}_{H, \beta \alpha}\right\rangle_{\vec{v}}+\frac{1}{m_{\beta}}\left\langle\vec{v}, \vec{J}_{G, \beta \alpha}-\vec{J}_{H, \alpha \beta}\right\rangle_{\vec{v}}=0
$$

which for arbitrary species implies:

$$
\left\langle\vec{v}, \vec{J}_{G, \alpha \beta}\right\rangle_{\vec{v}}=\left\langle\vec{v}, \vec{J}_{H, \beta \alpha}\right\rangle_{\vec{v}} .
$$

This symmetry can also be derived from the Landau formulation of the Fokker-Planck collision operator.

While the symmetry in Eq. 3.10 is always satisfied in the continuum, numerical truncation errors break it in the discrete. In order to enforce the relationship discretely, we introduce a set of parameters $\gamma_{\alpha \beta}$ in the collision operator as follows:

$$
C_{\alpha \beta}=\Gamma_{\alpha \beta} \nabla_{v} \cdot\left[\gamma_{\alpha \beta} \vec{J}_{G, \alpha \beta}-\frac{m_{\alpha}}{m_{\beta}} \vec{J}_{H, \alpha \beta}\right] .
$$

The parameters $\gamma_{\alpha \beta}$ are determined from Eq. 3.9 as:

$$
\gamma_{\alpha \beta}=\frac{\left\langle\vec{v}, \vec{J}_{H, \beta \alpha}\right\rangle_{\vec{v}}}{\left\langle\vec{v}, \vec{J}_{G, \alpha \beta}\right\rangle_{\vec{v}}} .
$$

Effectively, $\gamma_{\alpha \beta}$ acts as a nonlinear constraint that ensures that Eq. 3.9 is satisfied in the discrete (which in turn enforces discrete energy conservation). Per Eq. 3.10, $\gamma_{\alpha \beta}=1$ in the continuum. In the discrete, $\gamma_{\alpha \beta}=1+\mathcal{O}(\Delta)$, where $\Delta$ is a measure of the truncation error associated with the discretization of the flux. It is worthwhile to mention that, in principle, $\gamma_{\alpha \beta}$ can be applied to either contribution $\left(\vec{J}_{G}, \vec{J}_{H}\right)$ of the Fokker-Planck flux. We have chosen to apply $\gamma_{\alpha \beta}$ to the diffusion flux $\vec{J}_{G}$ for robustness and accuracy, since $\vec{J}_{G}$ is numerically less accurate to compute than the friction flux $\vec{J}_{H}$.

\subsection{Discrete Momentum Conservation Scheme}

Momentum conservation follows from Eq. 2.8:

$$
m_{\alpha}\left\langle\vec{v}, C_{\alpha \beta}\right\rangle_{\vec{v}}=-m_{\beta}\left\langle\vec{v}, C_{\beta \alpha}\right\rangle_{\vec{v}} .
$$

For a cylindrically symmetric system, only the parallel momentum component needs to be conserved (the perpendicular component integrates to zero by azimuthal symmetry). Following a similar procedure as before, we find:

$$
\left\langle 1, J_{\|, G, \alpha \beta}\right\rangle_{\vec{v}}=\left\langle 1, J_{\|, H, \beta \alpha}\right\rangle_{\vec{v}} .
$$

However, as written, Eq. 3.13 is ill-posed to define a discrete constraint. This is so because, in certain scenarios (e.g., PDF even in $v_{\|}$), each term may vanish independently. To prevent this, we decompose the parallel velocity integrals of the inner products in terms of partial integrals:

$$
\left\langle 1, J_{\|, G, \alpha \beta}\right\rangle_{u_{\|, \alpha \beta}}^{\infty}+\left\langle 1, J_{\|, G, \alpha \beta}\right\rangle_{-\infty}^{u_{\|, \alpha \beta}}=\left\langle 1, J_{\|, H, \beta \alpha}\right\rangle_{u_{\|, \alpha \beta}}^{\infty}+\left\langle 1, J_{\|, H, \beta \alpha}\right\rangle_{-\infty}^{u_{\|, \alpha \beta}},
$$

and derive the following symmetry:

$$
\left\langle 1, J_{\|, G, \alpha \beta}-J_{\|, H, \beta \alpha}\right\rangle_{u_{\|, \alpha \beta}}^{\infty}=-\left\langle 1, J_{\|, G, \alpha \beta}-J_{\|, H, \beta \alpha}\right\rangle_{-\infty}^{u_{\|, \alpha \beta}}
$$

where $\langle 1,(\cdot)\rangle_{a}^{b} \equiv \int_{-\infty}^{+\infty} v_{\perp} d v_{\perp} \int_{a}^{b}(\cdot) d v_{\|}$. This relationship is true for arbitrary $u_{\|, \alpha \beta}$. Here, $u_{\|, \alpha \beta}$ is chosen as the parallel center-of-mass fluid velocity for species $\alpha$ and $\beta$, which is defined as:

$$
u_{\|, \alpha \beta}=\frac{m_{\alpha}\left\langle v_{\|}, f_{\alpha}\right\rangle_{\vec{v}}+m_{\beta}\left\langle v_{\|}, f_{\beta}\right\rangle_{\vec{v}}}{m_{\alpha}\left\langle 1, f_{\alpha}\right\rangle_{\vec{v}}+m_{\beta}\left\langle 1, f_{\beta}\right\rangle_{\vec{v}}} .
$$


We note that the relationship in Eqn. (3.14) is satisfied for any arbitrary choice for $u_{\|, \alpha \beta}$. However, choosing the definition from Eq. (3.16) ensures that, numerically, each half integral is computed accurately.

As before, owing to discretization error, Eq. 3.15 is not strictly enforced discretely in general. In order to enforce it, we again add a constraint to the collision operator, which now reads:

$$
C_{\alpha \beta}=\Gamma_{\alpha \beta} \vec{\nabla}_{v} \cdot\left[\overleftrightarrow{\eta}_{\alpha \beta} \cdot \vec{J}_{G, \alpha \beta}-\frac{m_{\alpha}}{m_{\beta}} \vec{J}_{H, \alpha \beta}\right]
$$

Here, $\stackrel{\leftrightarrow}{\eta}_{\alpha \beta}$ is a tensor

$$
\stackrel{\leftrightarrow}{\eta}_{\alpha \beta}=\left[\begin{array}{cc}
\eta_{\|, \alpha \beta} & 0 \\
0 & 1
\end{array}\right]
$$

The coefficient $\eta_{\|, \alpha \beta}$ is of the form:

$$
\eta_{\|, \alpha \beta}= \begin{cases}\mathcal{E}_{\alpha \beta} & v_{\|} \geq u_{\|, \alpha \beta} \\ 1 & \text { otherwise }\end{cases}
$$

where $\mathcal{E}_{\alpha \beta}$ is found from Eq. 3.15 as:

$$
\mathcal{E}_{\alpha \beta}\left\langle 1, J_{\|, G, \alpha \beta}\right\rangle_{u_{\|, \alpha \beta}}^{\infty}+\left\langle 1, J_{\|, G, \alpha \beta}\right\rangle_{-\infty}^{u_{\|, \alpha \beta}}=\left\langle 1, J_{\|, H, \beta \alpha}\right\rangle_{u_{\|, \alpha \beta}}^{\infty}+\left\langle 1, J_{\|, H, \beta \alpha}\right\rangle_{-\infty}^{u_{\|, \alpha \beta}},
$$

and reads:

$$
\mathcal{E}_{\alpha \beta}=\frac{\left\langle 1, J_{\|, H, \beta \alpha}\right\rangle_{u_{\|, \alpha \beta}}^{\infty}+\left\langle 1, J_{\|, H, \beta \alpha}-J_{\|, G, \alpha \beta}\right\rangle_{-\infty}^{u_{\|, \alpha \beta}}}{\left\langle 1, J_{\|, G, \alpha \beta}\right\rangle_{u_{\|, \alpha \beta}}^{\infty}} .
$$

Per Eq. $3.15, \mathcal{E}_{\alpha \beta}=1$ in the continuum. Similarly to the discrete energy conservation scheme, $\eta_{\|, \alpha \beta}$ is applied to the diffusion flux rather than the friction flux for robustness and accuracy.

\subsection{Discrete Mass, Momentum, and Energy Conserving Scheme}

We combine the discrete mass, momentum, and energy conserving schemes to develop a simultaneous mass, momentum, and energy conserving discretization scheme. For this purpose, we propose the following formulation for the collision operator:

$$
C_{\alpha \beta}=\Gamma_{\alpha \beta} \vec{\nabla}_{v} \cdot\left[\left(\gamma_{\alpha \beta} \overleftrightarrow{I}+\stackrel{\leftrightarrow}{\epsilon}_{\alpha \beta}\right) \cdot \vec{J}_{G, \alpha \beta}-\frac{m_{\alpha}}{m_{\beta}} \vec{J}_{H, \alpha \beta}\right] .
$$

Here, as before, $\gamma_{\alpha \beta}$ is the energy conserving consistency term, while

$$
\stackrel{\leftrightarrow}{\epsilon}_{\alpha \beta}=\left[\begin{array}{cc}
\epsilon_{\|, \alpha \beta} & 0 \\
0 & 0
\end{array}\right]
$$

is the momentum conserving consistency term with $\epsilon_{\|, \alpha \beta}=\eta_{\|, \alpha \beta}-1$. The symmetries in Eqs. 3.10 and 3.15 read, after introducing $\gamma_{\alpha \beta}$ and $\stackrel{\leftrightarrow}{\epsilon}_{\alpha \beta}$ :

$$
\left\langle\vec{v},\left(\gamma_{\alpha \beta} \stackrel{\leftrightarrow}{I}+\stackrel{\leftrightarrow}{\epsilon}_{\alpha \beta}\right) \cdot \vec{J}_{G, \alpha \beta}\right\rangle_{\vec{v}}=\left\langle\vec{v}, \vec{J}_{H, \beta \alpha}\right\rangle_{\vec{v}}
$$

and

$$
\left\langle 1,\left(\gamma_{\alpha \beta}+\epsilon_{\|, \alpha \beta}\right) J_{\|, G, \alpha \beta}\right\rangle_{u_{\|, \alpha \beta}}^{\infty}+\left\langle 1, \gamma_{\alpha \beta} J_{\|, G, \alpha \beta}\right\rangle_{-\infty}^{u_{\|, \alpha \beta}}=\left\langle 1, J_{\|, H, \beta \alpha}\right\rangle_{u_{\|, \alpha \beta}}^{\infty}+\left\langle 1, J_{\|, H, \beta \alpha}\right\rangle_{-\infty}^{u_{\|, \alpha \beta}},
$$

which give:

$$
\gamma_{\alpha \beta}=\frac{\left\langle\vec{v}, \vec{J}_{H, \beta \alpha}\right\rangle_{\vec{v}}-\epsilon_{\|, \alpha \beta}\left\langle\vec{v}, \vec{J}_{\|, G, \alpha \beta}\right\rangle_{u_{\|, \alpha \beta}}^{+\infty}}{\left\langle\vec{v}, \vec{J}_{G, \alpha \beta}\right\rangle_{\vec{v}}}
$$


and:

$$
\epsilon_{\|, \alpha \beta}=\left\{\begin{array}{ll}
\frac{\left\langle 1, J_{\|, H, \beta \alpha}\right\rangle_{\vec{v}}-\gamma_{\alpha \beta}\left\langle 1, J_{\|, G, \alpha \beta}\right\rangle_{\vec{v}}}{\left\langle 1, J_{\|, G, \alpha \beta}\right\rangle_{u_{\|, \alpha \beta}^{+\infty}}^{+\infty}} & \text { if } v_{\|} \geq u_{\|, \alpha \beta} \\
0 & \text { otherwise }
\end{array} .\right.
$$

Equations 3.24 and 3.25 are a set of linear equations for $\epsilon_{\|, \alpha \beta}$ and $\gamma_{\alpha \beta}$, of trivial inversion. The definitions in these equations are valid for any definition of diffusion and friction fluxes, and therefore feature complete generality regardless of limiting, etc. Note that, in the continuum, $\gamma_{\alpha \beta}=1$ and $\epsilon_{\|, \alpha \beta}=0$. In the discrete, both parameters will deviate from these values by a small amount due to truncation error.

We remark that the definition of the conservation constants $\left(\gamma_{\alpha \beta}\right.$ and $\left.\epsilon_{\|, \alpha \beta}\right)$ has been derived in the continuum. No particular discretization has been assumed, and the scheme will hold in very general cases (including that of multiple velocity space grids [42]). Additionally, the scheme can be generalized to $3 \mathrm{~V}$, requiring a trivial nonlinear solve for four conservation constants (one $\gamma_{\alpha \beta}$ and three $\epsilon_{\alpha \beta}$ for each component of velocity space). It is also worthwhile to note that this general approach of introducing a constraint coefficient to enslave the truncation error of the conservation properties into the discretization was first demonstrated in Ref. [43] in the context of the Vlasov-Ampère system.

\section{Implicit temporal discretization and nonlinear solver strategy}

When using explicit schemes, the stiff explicit time-step size, $\Delta t_{\text {exp }}$, must be resolved for numerical stability reasons. The explicit time step can be estimated as:

$$
\Delta t_{\text {exp }} \approx \nu_{c o l}^{-1} \frac{\Delta v^{2}}{v_{t h}^{2}}
$$

where $v_{t h}=\sqrt{\frac{2 T}{m}}$ is the thermal velocity, and $\nu_{c o l}^{-1}=\frac{3}{4} \frac{\sqrt{m}}{\sqrt{2 \pi}} \frac{T^{3 / 2}}{Z^{4} n e^{4} \Lambda}$ is the inverse thermal collision frequency. Since $\Delta v \ll v_{t h}$, it follows that $\Delta t_{\text {exp }} \nu_{c o l} \ll 1$, i.e., explicit methods must resolve the fastest collisional time scale at any point in time, and can therefore be very inefficient when much longer dynamical time scales are of interest.

Implicit methods remove the time-step stability constraint, and have the potential of following slower dynamical time scales of interest at much reduced computational cost. In this study, we focus on fully implicit, nonlinear temporal discretizations. Fully implicit schemes, when combined with strict conservation properties, guarantee that the solution is asymptotic-preserving in the limit of strong collisionality to $\mathcal{O}\left(\epsilon^{2}\right)$, with $\epsilon=\nu_{c o l}^{-1} / \tau_{0} \ll 1$ a measure of the collisional time scale normalized to the characteristic time scale of interest. To show this, we consider the following model for the Fokker-Planck equation:

$$
\partial_{t} f=\frac{1}{\epsilon} Q(f, f)
$$

with $Q\left(f_{M}, f_{M}\right)=0$ (i.e., the Maxwellian is the equilibrium solution). We consider a first-order backward Euler integration approach for simplicity, which gives:

$$
\frac{f^{n+1}-f^{n}}{\Delta t}=\frac{1}{\epsilon} Q\left(f^{n+1}, f^{n+1}\right) .
$$

In the small Knudsen number limit, we consider solutions of the form $f=f_{M}+\epsilon f_{1}$. Introducing this ansatz in the equation above, noting that, owing to strict conservation properties, the Maxwellian is the equilibrium solution numerically as well (i.e., $f_{M}^{n+1}=f_{M}^{n}$ ), and noting that $Q\left(f^{n+1}, f^{n+1}\right)=\left.\epsilon \frac{\partial Q}{\partial f}\right|_{f_{M}} f_{1}^{n+1}+\mathcal{O}\left(\epsilon^{2}\right)$, yields:

$$
\left[\frac{\epsilon}{\Delta t} \mathbb{I}-\frac{\partial Q}{\partial f}+\mathcal{O}(\epsilon)\right] f_{1}^{n+1}=\frac{\epsilon}{\Delta t} f_{1}^{n},
$$

which gives:

$$
\frac{\partial Q}{\partial f} f_{1}^{n+1}=\mathcal{O}(\epsilon) \Rightarrow f_{1}^{n+1}=\mathcal{O}(\epsilon) .
$$

It follows that $f=f_{M}+\mathcal{O}\left(\epsilon^{2}\right)$ for $\epsilon \ll 1$. 
In this study, we employ second-order backward differentiation formulas (BDF2) [44]. For a given timelevel, $n$, the Fokker-Planck equation is time-discretized as:

$$
\frac{c_{n+1} f^{n+1}+c_{n} f^{n}+c_{n-1} f^{n-1}}{\Delta t^{n+1}}-\sum_{\beta}^{N_{s}} C_{\alpha \beta}\left(f_{\alpha}^{n+1}, f_{\beta}^{n+1}\right)=0,
$$

where the coefficients $c_{n+1}, c_{n}$, and $c_{n-1}$ are the temporal weights for the BDF2 method, and $\Delta t^{n+1}$ is the current time-step size. For a uniform time step, $c_{n+1}=3 / 2, c_{n}=-2, c_{n-1}=1 / 2$. For variable time steps, we have:

$$
c_{n+1}=1+\gamma, c_{n}=-\frac{1}{1-\gamma}, c_{n-1}=\frac{\gamma^{2}}{1-\gamma},
$$

with $\gamma=\frac{\Delta t_{n+1}}{\Delta t_{n+1}+\Delta t_{n}}<1$. We have the capability of adapting the time step based on the performance of the Newton iteration, although this is not employed in this study.

Upon discretization, implicit methods for the Fokker-Planck result in a large, nonlinear set of algebraic equations and nonlinear constraints (to enforce conservation properties), the solution of which requires a clever nonlinear solver strategy. We rely on Jacobian-free Newton-Krylov methods (JFNK) [45], efficiently preconditioned with multigrid methods, for this task. We briefly introduce JFNK and our preconditioning strategy in the next subsections.

\subsection{The Jacobian-Free Newton-Krylov Method}

Jacobian-free Newton-Krylov methods [46] attempt to find the root of a nonlinear residual $\overrightarrow{\mathbb{R}}(\vec{x})=0$ iteratively by a Newton-Raphson procedure. Upon linearization, the Newton system at the $k^{\text {th }}$ nonlinear iteration,

$$
\mathbb{J}^{k} \delta \vec{x}^{k}=-\overrightarrow{\mathbb{R}}^{k},
$$

is solved for the Newton update, $\delta \vec{x}^{k}$, using a Krylov subspace method [46] (flexible GMRES [47] is used in this study, to accommodate the nonlinear enslavement of the Rosenbluth potentials, as described below). Here, $\mathbb{J}=\frac{\partial \overrightarrow{\mathbb{R}}}{\partial \vec{x}}$ is the Jacobian matrix, $\overrightarrow{\mathbb{R}}$ is the nonlinear residual vector,

$$
\overrightarrow{\mathbb{R}}=\left\{\begin{array}{l}
\overrightarrow{\mathbb{R}}_{f} \\
\overrightarrow{\mathbb{R}}_{H} \\
\overrightarrow{\mathbb{R}}_{G} \\
\overrightarrow{\mathbb{R}}_{\gamma} \\
\overrightarrow{\mathbb{R}}_{\epsilon}
\end{array}\right\}
$$

where,

$$
\begin{gathered}
\overrightarrow{\mathbb{R}}_{f}=\left[\begin{array}{c}
\partial_{t} f_{1}-\sum_{\beta}^{N_{s}} C_{1 \beta} \\
\vdots \\
\partial_{t} f_{N_{s}}-\sum_{\beta}^{N_{s}} C_{N_{s} \beta}
\end{array}\right], \overrightarrow{\mathbb{R}}_{H}=\left[\begin{array}{c}
\nabla_{v}^{2} H_{1}+8 \pi f_{1} \\
\vdots \\
\nabla_{v}^{2} H_{N_{s}}+8 \pi f_{N_{s}}
\end{array}\right], \\
\overrightarrow{\mathbb{R}}_{G}=\left[\begin{array}{c}
\nabla_{v}^{2} G_{1}-H_{1} \\
\vdots \\
\nabla_{v}^{2} G_{N_{s}}-H_{N_{s}}
\end{array}\right], \\
\overrightarrow{\mathbb{R}}_{\gamma}=\left[\left\langle\vec{v},\left(\gamma_{\alpha \beta} \stackrel{\leftrightarrow}{I}+\stackrel{\leftrightarrow}{\epsilon}_{\alpha \beta}\right) \cdot \vec{J}_{G, \alpha \beta}\right\rangle_{\vec{v}}-\left\langle\vec{v}, \vec{J}_{H, \alpha \beta}\right\rangle_{\vec{v}}\right] \text { for } \alpha, \beta=1, \ldots, N_{s}, \\
\overrightarrow{\mathbb{R}}_{\epsilon}=\left[\left\langle 1,\left(\gamma_{\alpha \beta}+\epsilon_{||, \alpha \beta}\right) J_{||, G, \alpha \beta}\right\rangle_{\vec{v}}-\left\langle 1, J_{\|, H, \alpha \beta}\right\rangle_{\vec{v}}\right] \text { for } \alpha, \beta=1, \ldots, N_{s},
\end{gathered}
$$

and $\vec{x}$ is the solution vector, 


$$
\begin{gathered}
\vec{x}=\left\{\begin{array}{c}
\vec{f} \\
\vec{H} \\
\vec{G} \\
\vec{\gamma} \\
\vec{\epsilon}
\end{array}\right\}, \vec{f}=\left[\begin{array}{c}
f_{1} \\
\vdots \\
f_{N_{s}}
\end{array}\right], \vec{H}=\left[\begin{array}{c}
H_{1} \\
\vdots \\
H_{N_{s}}
\end{array}\right], \vec{G}=\left[\begin{array}{c}
G_{1} \\
\vdots \\
G_{N_{s}}
\end{array}\right], \\
\vec{\gamma}=\left[\gamma_{\alpha \beta}\right], \vec{\epsilon}=\left[\epsilon_{\|, \alpha \beta}\right] \text { for } \alpha, \beta=1, \ldots, N_{s},
\end{gathered}
$$

which we update from the Newton iteration as,

$$
\vec{x}^{k+1}=\vec{x}^{k}+\delta \vec{x}^{k} .
$$

The Newton iteration is continued until:

$$
\left\|\overrightarrow{\mathbb{R}}\left(\vec{x}^{(k)}\right)\right\|_{2}<\epsilon_{a}+\epsilon_{r}\left\|\overrightarrow{\mathbb{R}}\left(\vec{x}^{(0)}\right)\right\|_{2}=\epsilon_{t} .
$$

Here, $\|\cdot\|$ is the $L_{2}$-norm, $\epsilon_{a}=\sqrt{N} \times 10^{-15}$ (with $N$ the total number of degrees of freedom) is an absolute tolerance to avoid converging below round-off, $\epsilon_{r}$ is the Newton relative convergence tolerance (set to $10^{-4}$ in this study), and $\overrightarrow{\mathbb{R}}\left(\vec{x}^{(0)}\right)$ is the initial residual.

In the Krylov iteration, only the action of the Jacobian on the Krylov vector, $\vec{v}_{k}$, is required. Such matrix-vector products can be implemented Jacobian-free using the Gateaux derivative:

$$
\mathbb{J} \vec{v}_{k} \approx \frac{\mathbb{R}\left(\vec{x}+\epsilon \vec{v}_{k}\right)-\mathbb{R}(\vec{x})}{\epsilon},
$$

where in practice a small but finite $\epsilon$ is employed (p. 79 in [46]). Convergence for the Krylov solver is determined by an inexact Newton method [48], which adapts the Krylov linear convergence tolerance at the $k^{\text {th }}$ Newton iteration, $\zeta^{(k)}$, as [48]:

$$
\begin{gathered}
\zeta^{A(k)}=\gamma\left(\frac{\left\|\overrightarrow{\mathbb{R}}\left(\vec{x}^{(k)}\right)\right\|_{2}}{\left\|\overrightarrow{\mathbb{R}}\left(\vec{x}^{(k-1)}\right)\right\|_{2}}\right)^{\alpha}, \\
\zeta^{B(k)}=\min \left[\zeta_{\max }, \max \left(\zeta^{A(k)}, \gamma\left(\zeta^{(k-1)}\right)^{\alpha}\right)\right], \\
\zeta^{(k)}=\min \left[\zeta_{\max }, \max \left(\zeta^{B(k)}, \gamma \frac{\epsilon_{t}}{\left\|\overrightarrow{\mathbb{R}}\left(\vec{x}^{(k)}\right)\right\|_{2}}\right)\right]
\end{gathered}
$$

and iterated until:

$$
\frac{\left\|\rrbracket^{k} \delta \vec{x}^{k}-\mathbb{R}^{k}\right\|}{\left\|\mathbb{R}^{k}\right\|}<\zeta^{(k)}
$$

In this study, $\alpha=1.5, \gamma=0.9$, and $\zeta_{\max }=0.8$.

\subsection{Nonlinear elimination of Rosenbluth potentials and nonlinear conservation constraints}

As defined, for an $N_{v}=N_{\|} N_{\perp}$ mesh, the dimension of the nonlinear residual scales as $N_{v} \times 3 N_{s}+2 N_{s}^{2}$. To reduce storage (particularly when one or more spatial dimensions are considered), to facilitate a practical implementation, and to simplify preconditioning, it is advantageous to simplify the nonlinear residual by nonlinear elimination.

Nonlinear elimination has seen applications in both collisional [32] and collisionless kinetic plasmas [49, 50], thermal radiation transport [51], and neutron transport [52, 53]. The basic idea behind nonlinear elimination is to enslave one or more nonlinear variables within a residual function evaluation for another nonlinear variable to effectively reduce the nonlinear system size and/or simplify implementation. In this study, we follow Refs. [32] and enslave the inversion of the Rosenbluth potentials into the residual function 


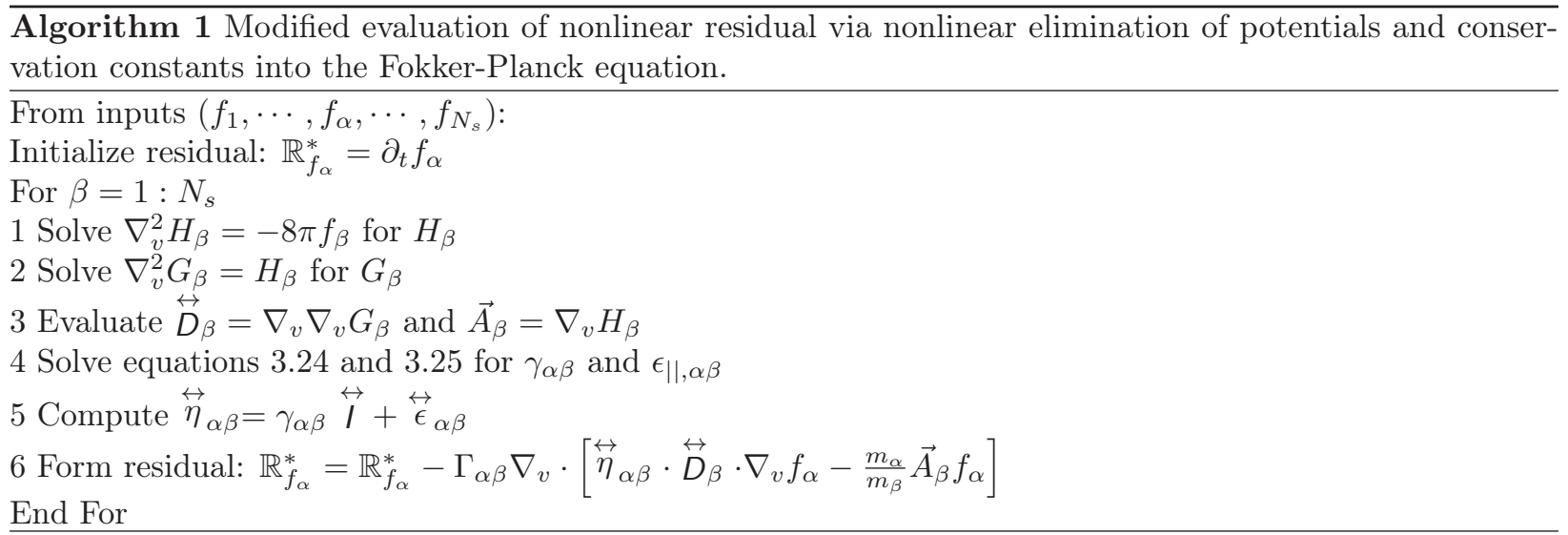

evaluation for the Fokker-Planck equation (i.e., we perform the Poisson inversions directly in the nonlinear residual). Furthermore, we also enslave the nonlinear conservation constraints by inverting the associated linear system directly, and replacing them into the Fokker-Planck collision operator at flux-assembly time. The resulting system size scales as $N_{v} \times N_{s}$, and has the advantage that 1) conservation constraints are enforced at every residual evaluation during the nonlinear iteration, and 2) preconditioning will be greatly simplified (Sec. 4.3).

Formally, the nonlinear elimination procedure can be expressed as follows. We note first that assembling the discrete $(\alpha, \beta)$ collision operator requires the Rosenbluth potentials, $H_{\beta}, G_{\beta}$, and the conservation constants $\gamma_{\alpha \beta}$ and $\epsilon_{\|, \alpha \beta}$, which in turn are all functionals of the distribution functions $f_{\alpha}, f_{\beta}$. Therefore:

$$
C_{\alpha \beta}\left(f_{\alpha}, H_{\beta}, G_{\beta}, \gamma_{\alpha \beta}, \epsilon_{\|, \alpha \beta}\right)=C_{\alpha \beta}\left(f_{\alpha}, H_{\beta}\left[f_{\beta}\right], G_{\beta}\left[f_{\beta}\right], \gamma_{\alpha \beta}\left[f_{\alpha}, f_{\beta}\right], \epsilon_{\|, \alpha \beta}\left[f_{\alpha}, f_{\beta}\right]\right)=\widetilde{C}_{\alpha \beta}\left(f_{\alpha}, f_{\beta}\right),
$$

allowing a new residual, $\overrightarrow{\mathbb{R}}^{*}$, to be formulated as:

$$
\overrightarrow{\mathbb{R}}(\vec{x})=\overrightarrow{\mathbb{R}}^{*}\left(\vec{x}^{*}\right)=0,
$$

where

$$
\overrightarrow{\mathbb{R}}^{*}=\left\{\begin{array}{c}
\mathbb{R}_{f_{1}}^{*} \\
\vdots \\
\mathbb{R}_{f_{N_{s}}}^{*}
\end{array}\right\}, \vec{x}^{*}=\left\{\begin{array}{c}
f_{1} \\
\vdots \\
f_{N_{s}}
\end{array}\right\},
$$

with $\mathbb{R}_{f_{\alpha}}^{*}$ evaluated by Algorithm 1.

The required Rosenbluth Poisson solves in the residual are performed by multigrid-preconditioned conjugate gradient (CG) method. For the multigrid preconditioner, we employ 1 V-cycle of classical multigrid, smoothed with 4 passes of damped Jacobi (with damping constant 0.7) [35]. We employ linear restriction and prolongation. This setup results in convergence of the Poisson systems to a relative tolerance of $10^{-8}$ in $\mathcal{O}(10)$ CG iterations, independent of mesh refinement. Far-field boundary conditions are imposed for the potentials $G_{\beta}$ and $H_{\beta}$ using the Green's function solution, as described in Ref. [34]. A priori, if far-field boundary values are evaluated at all ghost cells, the computational cost will scale as $\left(N_{\|}+2 N_{\perp}\right) N_{\|} N_{\perp} \sim \mathcal{O}\left(N_{v}^{3 / 2}\right)$, which is non-optimal. To make it optimal $\left[\mathcal{O}\left(N_{v}\right)\right]$, we evaluate far-field boundary values only at a fixed number of boundary points $(\sim 20)$, which are then used to fill all ghost cells at the boundary by interpolation (Fig. 4.1). This process is justified by the smoothness of the potentials, and the assumption that $f$ has compact support in the computational domain.

\subsection{Preconditioning: Block Diagonalization and Multigrid}

JFNK is a Krylov-based nonlinear solver, and preconditioning is key for performance. The Jacobian system for the enslaved nonlinear residual reads: 


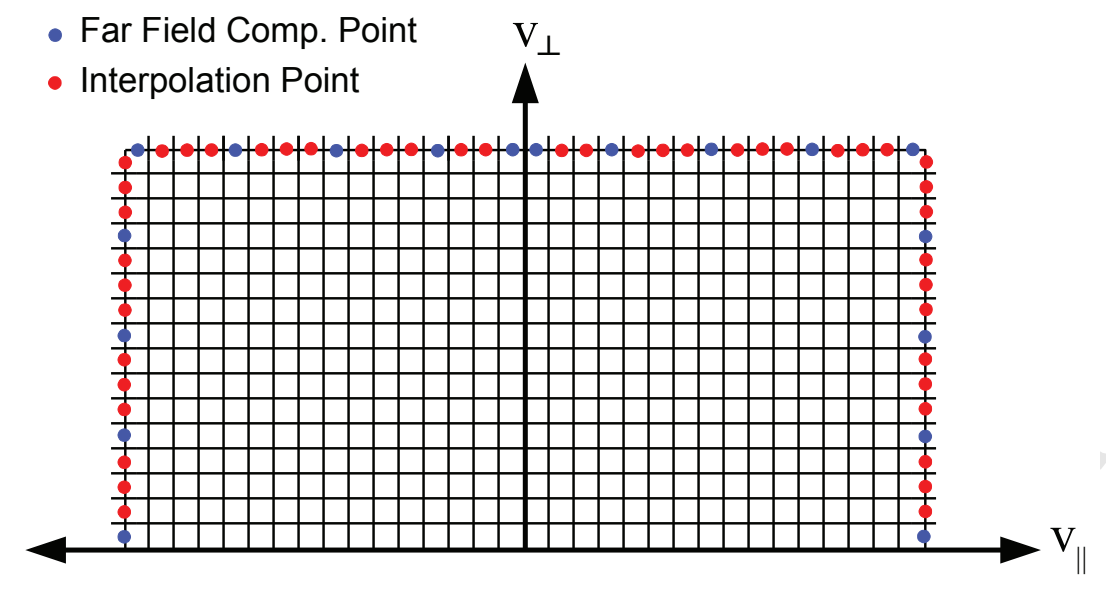

Figure 4.1: Interpolation of specified far-field boundary compute points to neighbors.

$$
\mathbb{J}^{*} \delta \vec{x}^{*}=-\overrightarrow{\mathbb{R}}^{*},
$$

where $\mathbb{J}^{*}=\frac{\partial \overrightarrow{\mathbb{R}}^{*}}{\partial \vec{x}^{*}}$. We employ a right preconditioning strategy,

$$
\mathbb{J}^{*} \mathbb{P}^{-1} \delta \vec{y}=-\overrightarrow{\mathbb{R}}^{*},
$$

where the solution is found upon convergence as:

$$
\delta \vec{x}^{*}=\mathbb{P}^{-1} \delta \vec{y},
$$

and $\mathbb{P}^{-1}$ is the preconditioning operator. In the context of JFNK, preconditioning is performed by modifying the matrix-vector product as:

$$
\mathbb{J}^{*} \mathbb{P}^{-1} v_{k} \approx \frac{\overrightarrow{\mathbb{R}}^{*}\left(\vec{x}^{*}+\epsilon \mathbb{P}^{-1} \vec{v}_{k}\right)-\overrightarrow{\mathbb{R}}^{*}\left(\vec{x}^{*}\right)}{\epsilon} .
$$

For preconditioning, we lag the transport coefficients $\stackrel{\leftrightarrow}{D}_{\beta}$ and $\vec{A}_{\beta}$ to the previous Newton iteration. It follows that the preconditioning operator for species $\alpha$ is:

$$
\mathbb{P}_{\alpha}^{k} \circ=\partial_{t} \circ-\nabla_{v} \cdot[\underbrace{\left(\sum_{\beta}^{N_{s}} \Gamma_{\alpha \beta} \stackrel{\leftrightarrow}{D}_{\beta}^{k-1}\right)}_{\bar{D}_{\alpha}} \cdot \nabla_{v} \circ-m_{\alpha} \underbrace{\left(\sum_{\beta}^{N_{s}} \frac{\Gamma_{\alpha \beta}}{m_{\beta}} \vec{A}_{\beta}^{k-1}\right)}_{\vec{A}_{\alpha}} \circ],
$$

where the transport coefficients, $\stackrel{\leftrightarrow}{D}_{\alpha}$ and $\vec{A}_{\alpha}$ are known. Thus, lagging the transport coefficients effectively block-diagonalizes the Jacobian matrix, eliminating the cross-species coupling and greatly reducing the cost of the preconditioner inversion.

Each block in the preconditioner becomes a simple advection-diffusion equation in velocity space, which can be dealt with effectively with multigrid methods [32]. The advection operator is upwinded for robustness. Here, we employ $3 \mathrm{~V}$-cycles of classical multigrid, smoothed with 3 passes of damped Jacobi (with damping constant 0.7) [35]. We employ agglomeration for restriction, and second-order prolongation. The effectiveness of the preconditioner with respect to time-step size and grid refinement will be demonstrated in Sec. 5 . 


\section{Numerical results}

We have studied the new conservative discretization scheme for the Rosenbluth-Fokker-Planck operator by performing a suite of numerical tests. These tests, presented next in increasing order of complexity, are designed to challenge the solver in various ways, and to demonstrate the advertised properties of the scheme.

Before we introduce the results, an important comment is in order. The conservation properties in our scheme are enforced by nonlinear constraints. These nonlinear constraints are enforced in every residual evaluation, and thus they hold for arbitrary nonlinear convergence tolerances provided no preconditioning is employed. The preconditioner does not enforce these properties explicitly, and thus, with preconditioning, these properties will only hold to the nonlinear tolerance level. With right preconditioning (the choice here), the error is introduced in the last application of the preconditioner (Eq. 4.1), when undoing the change of variables. This will be borne out by the numerical experiments that follow.

\subsection{Single species maintaining thermal equilibrium}

We demonstrate the importance of discrete conservation properties on a single species problem maintaining a thermal equilibrium. The initial condition is set to a drifting Maxwellian distribution function,

$$
f\left(v_{\|}, v_{\perp}, t=0\right)=\frac{n_{0}}{\left(2 \pi T_{0} / m\right)^{3 / 2}} \exp \left[-\frac{m\left(\left[v_{\|}-u_{0}\right]^{2}+v_{\perp}^{2}\right)}{2 T_{0}}\right],
$$

where the initial density, fluid velocity, and temperature are set to $n_{0}=1, u_{0}=0$, and $T_{0}=0.5$, respectively, and $m=1$ and $Z=1$. The velocity space domain is $v_{\|} \in\left[-10 v_{t h, 0}, 10 v_{t h, 0}\right]$ and $v_{\perp} \in\left[0,10 v_{t h, 0}\right]$, where $v_{t h, 0}=\sqrt{2 T_{0} / m}$ is the initial thermal velocity. We discretize the domain with a $128 \times 64$ mesh. A maximum time-step size of $\Delta t_{\max }=1 \times 10^{-2} \approx \tau$ is used, where $\tau=\frac{3}{4} \frac{\sqrt{m}}{\sqrt{2 \pi}} \frac{T^{3 / 2}}{Z^{4} n_{0} e^{4} \Lambda}$ is the species self-collision time-scale with $n_{0}=1, Z=1, e=1, m=1, T=0.5$, and $\Lambda=10$.

Numerical results with and without discrete conservation properties are shown in Fig. 5.1. The discrete mass conservation,

$$
\Delta M^{n}=\left|M^{n}-M^{0}\right|
$$

parallel momentum conservation,

$$
\Delta I^{n}=\left|I^{n}-I^{0}\right|
$$

energy conservation,

$$
\Delta U^{n}=\left|U^{n}-U^{0}\right|
$$

and entropy conservation,

$$
\Delta S^{n}=S^{n}-S^{0},
$$

are depicted. Here, $M^{n}=\sum_{\alpha}^{N_{s}} m_{\alpha}\left\langle 1, f_{\alpha}^{n}\right\rangle_{\vec{v}}, I^{n}=\sum_{\alpha}^{N_{s}} m_{\alpha}\left\langle v_{\|}, f_{\alpha}^{n}\right\rangle_{\vec{v}}, U^{n}=\sum_{\alpha}^{N_{s}} \frac{m_{\alpha}}{2}\left\langle v^{2}, f_{\alpha}^{n}\right\rangle_{\vec{v}}, S^{n}=$ $-\sum_{\alpha}\left\langle\ln \left|f_{\alpha}^{n}\right|, f_{\alpha}^{n}\right\rangle_{\vec{v}}$ are the total mass, momentum, energy, and entropy of the system, and superscripts denote the time level. As can be seen, without conservation, the total energy change grows and the entropy (unphysically) increases indefinitely. (Mass and parallel momentum are still well conserved due to boundary conditions and symmetry, respectively). With the fully conservative formulation, all quantities are conserved down to the relative nonlinear tolerance $\left(\epsilon_{r}=10^{-4}\right)$ and the solution is held at a numerical equilibrium. Figure 5.1 also shows the impact of conservation in the conservation of entropy (for this test, since the initial condition begins with the equilibrium solution, entropy should be conserved). Clearly, while the conservative scheme is able to keep the entropy deviation small and constant (except for a fast transient in the beginning when the analytical Maxwellian relaxes to a numerical one), the non-conservative one systematically increases entropy. The lack of energy conservation leads to numerical cooling, as can be appreciated in Fig. 5.2, which shows the temporal evolution of the temperature, $T=\frac{m}{3} \frac{\left\langle|\vec{v}-\vec{u}|^{2}, f\right\rangle_{\vec{v}}}{\langle 1, f\rangle_{\vec{v}}}$. 

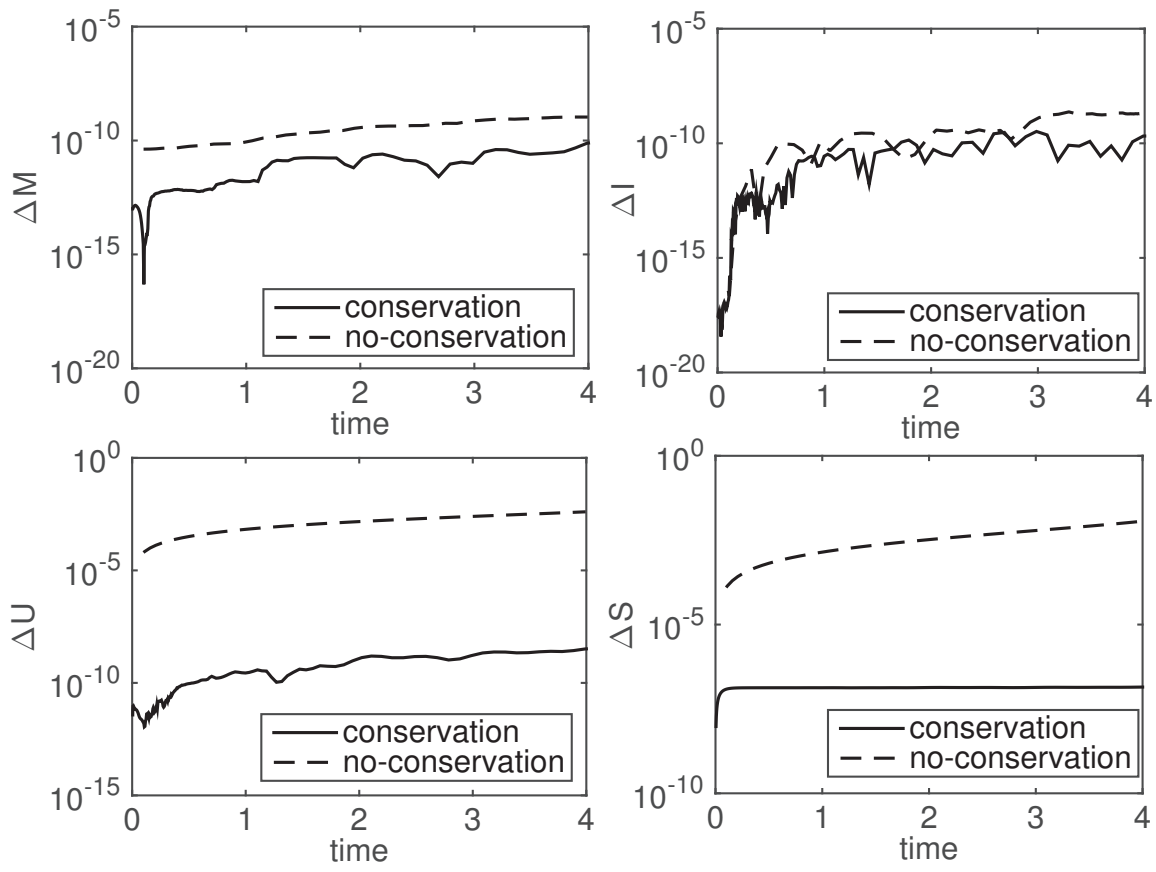

Figure 5.1: Single species in thermal equilibrium: Demonstration of conservation properties with and without discretely conservative formulation.

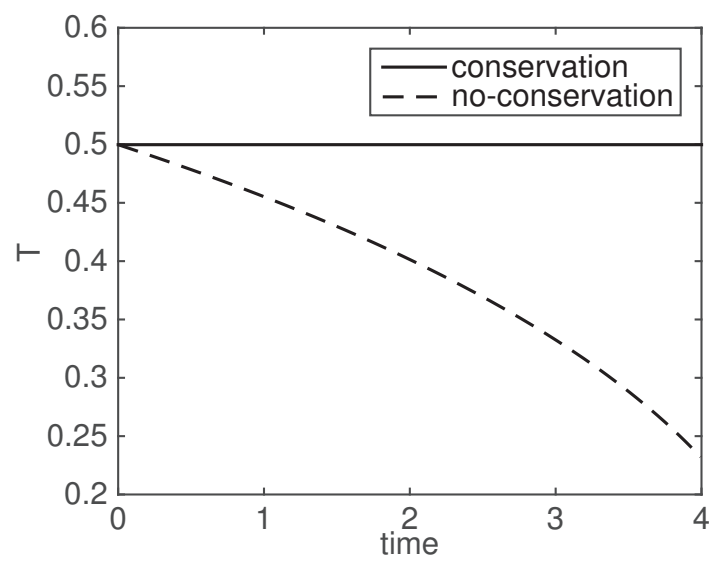

Figure 5.2: Single species in thermal equilibrium: Comparison of temperature evolution with and without discretely conservative formulation. 


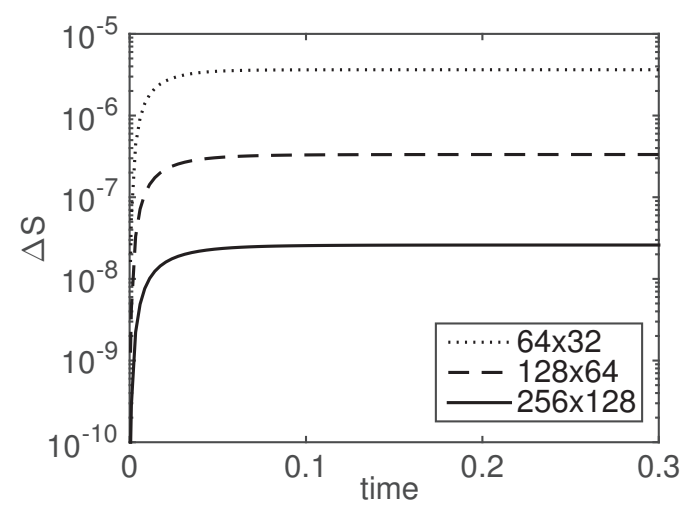

Figure 5.3: Single species in thermal equilibrium: Numerical entropy production as a function of grid resolution.

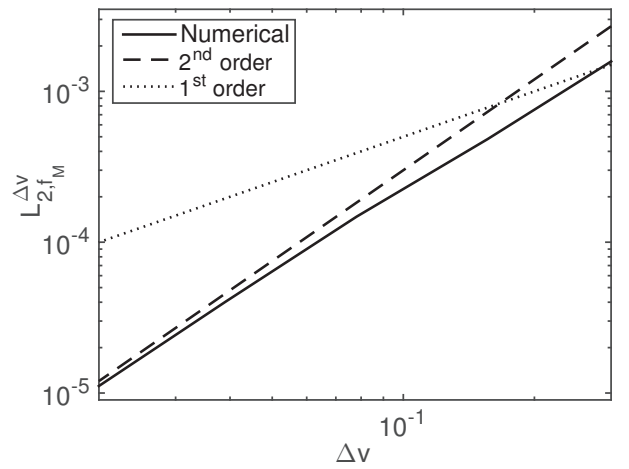

Figure 5.4: Single species in thermal equilibrium: Demonstration of second-order convergence of the velocity-space discretization scheme.

Figure 5.3 depicts the total entropy production in the system with the conservative scheme as a function of grid size for this test problem, demonstrating that the entropy increases with time during relaxation to the numerical Maxwellian, and that entropy error decreases with grid refinement. To demonstrate the order of accuracy of the velocity-space discretization, we compute the $L_{2}$-norm of the difference between the steady-state solution and the Maxwellian distribution function,

$$
L_{2}^{\Delta v}=\sqrt{\left\langle f_{j, k}-f_{M, j, k}, f_{j, k}-f_{M, j, k}\right\rangle_{\vec{v}}}
$$

for various mesh sizes $\Delta v$, and the result is shown in Figure 5.4. Second-order convergence is seen with the method with a slight degradation for coarser meshes. This is due to the SMART and the MC tensor-diffusion limiters, which resort to first-order discretizations to preserve positivity.

The impact of the preconditioning step on the overall conservation properties of the algorithm is demonstrated in Fig. 5.5. These tests have been performed with and without preconditioning with a small time-step size for a short time period. As is clearly demonstrated, without preconditioning, conservation in all quantities is enforced to numerical round-off. Preconditioning introduces an error of the order of nonlinear convergence tolerance. Despite this error, preconditioning is essential for the efficiency of the algorithm, as will be demonstrated shortly.

In regards to positivity, Fig. 5.6 depicts the magnitude of negative values of the distribution function after a single time-step for various nonlinear tolerances, demonstrating that the discretization scheme preserves positivity of the Maxwellian solution within numerical roundoff for arbitrary nonlinear tolerances.

It is important to point out that the superior properties of the velocity-space discretization proposed here are not limited to implicit timestepping, and in fact survive for explicit methods as long as the nonlinear residual is used without modifications to drive the explicit temporal scheme. To demonstrate this, we have implemented a second-order explicit Runge-Kutta scheme [54]. The conservation properties are shown in 

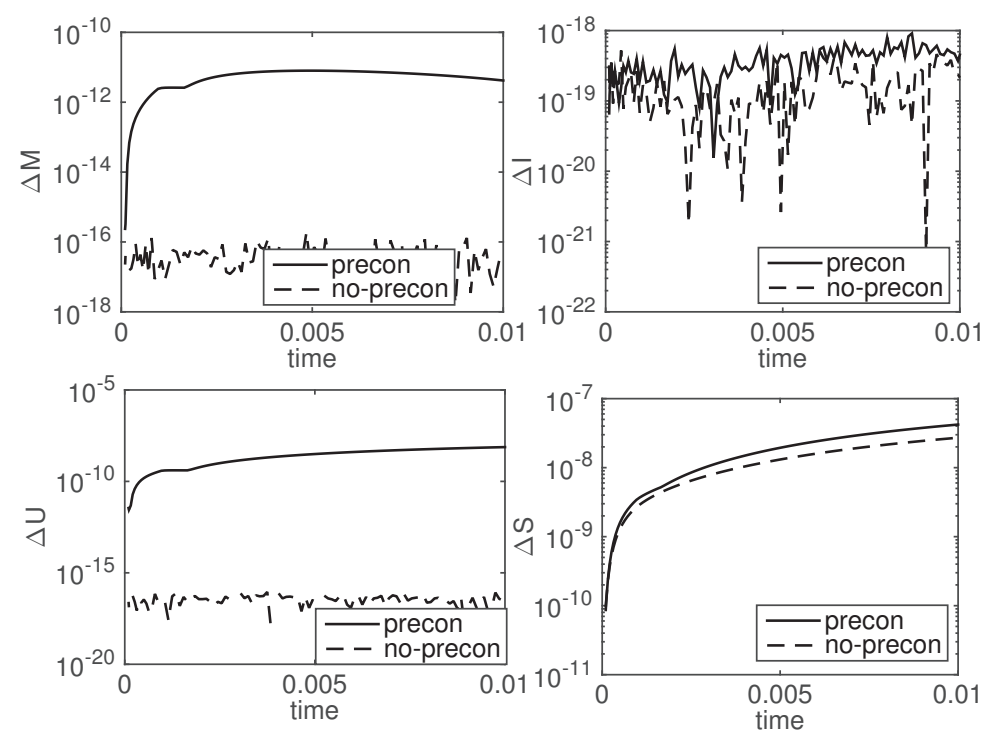

Figure 5.5: Single species in thermal equilibrium: Impact of preconditioning on conservation errors.

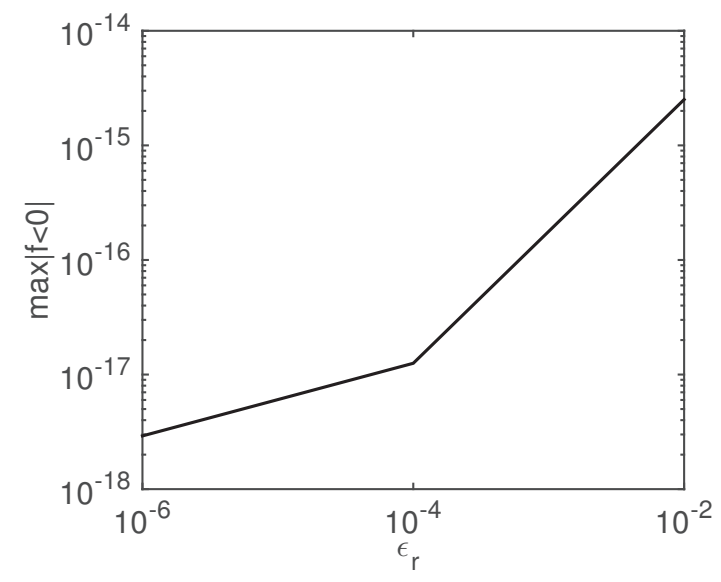

Figure 5.6: Single species in thermal equilibrium: Impact of nonlinear tolerance on positivity. The magnitude of negative values of the distribution function are shown after a single time-step. 

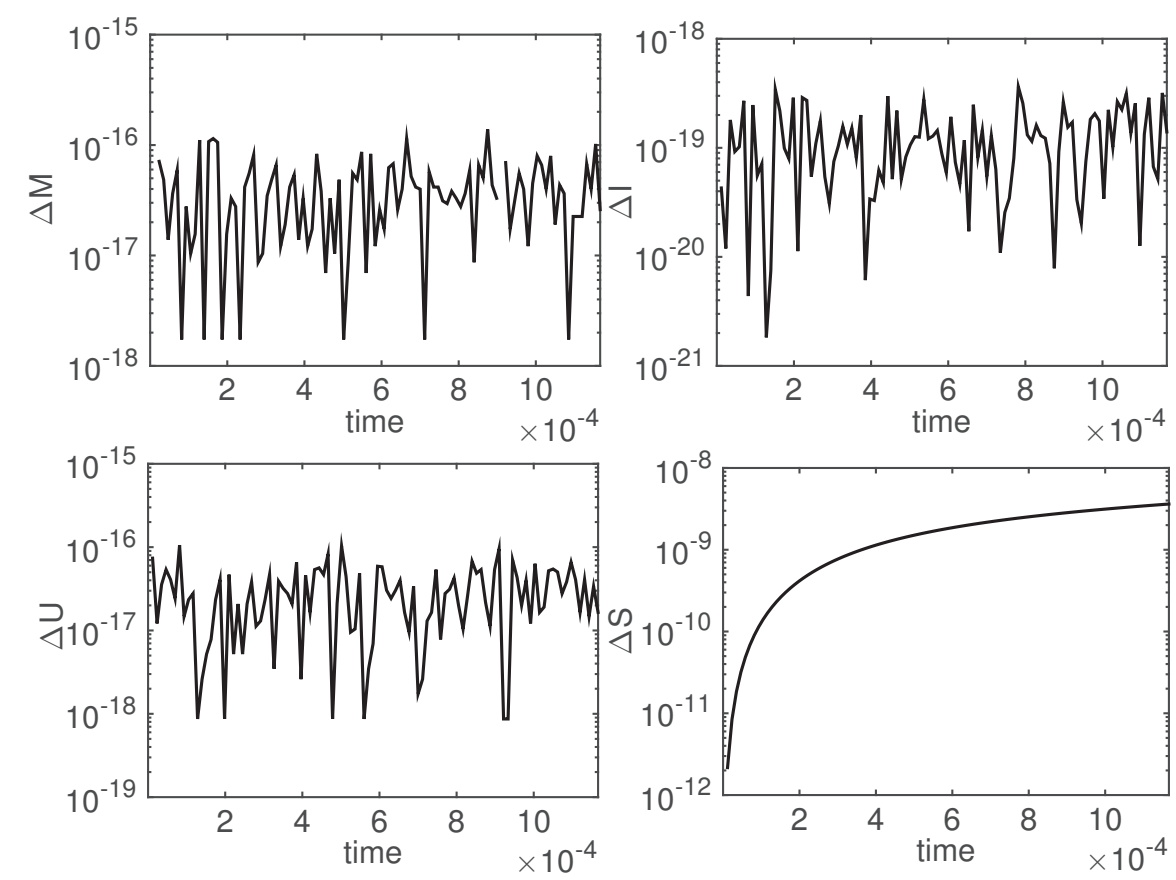

Figure 5.7: Single species in thermal equilibrium: Conservation properties are enforced for explicit schemes as well. The grid resolution considered is $N_{v}=128 \times 64$.

Figure 5.7 for a short time period, demonstrating that they are enforced to numerical round off. Entropy increases monotonically, as expected.

\subsection{Single species random distribution equilibration}

For the second test case, we employ a random distribution function relaxing to a Maxwellian. This test is particularly challenging for the positivity-preserving aspect of our algorithm, as the initial condition features grid-scale gradient length-scales. In particular, the distribution function is initialized as a specified number of Maxwellian distribution functions with random density, $n_{l}$, fluid velocities, $u_{\|, l}, u_{\perp, l}$, and temperature, $T_{l}$, as follows:

$$
f\left(v_{\|}, v_{\perp}, t=0\right)=\sum_{l}^{N_{m}} \frac{n_{l}}{\left(2 \pi T_{l} / m\right)^{3 / 2}} \exp \left[-\frac{m\left(\left[\left(v_{\|}-u_{\|, l}\right)^{2}+\left(v_{\perp}-u_{\perp, l}\right)^{2}\right]\right)}{2 T_{l}}\right],
$$

where $N_{m}=30$ is the number of Maxwellians, and:

$$
\begin{aligned}
n_{l} & =0.1 \operatorname{rand}() \\
u_{\|, l} & =3.5[1-2 \operatorname{rand}()] \\
u_{\perp, l} & =3.5 \operatorname{rand}() \\
T_{l} & =0.00125+(0.02-0.00125) \operatorname{rand}()
\end{aligned}
$$

where $\operatorname{rand}()$ is a random number generator. For this test problem, we choose $m=1, Z=1$, and use $\Delta t_{\max }=1 \times 10^{-3}$ and (unless stated otherwise) a relative nonlinear convergence tolerance of $\epsilon_{r}=10^{-6}$. For reference, with a random-number seed of 283 , we obtain $n=66.4$ and $T=3.2$, which give $\tau=2.5 \times 10^{-3}$.

In Fig. 5.8, we show the conservation properties and entropy as a function of time for various $\epsilon_{r}$. As expected, all quantities are conserved down to or near the nonlinear convergence tolerance. Also, entropy physically grows and saturates as the distribution equilibrates. In Fig. 5.9, the distribution function is shown at $t=0, t=2 \times 10^{-4}$, and $t=5 \times 10^{-2}$. We see that the initial random distribution features very sharp gradients, and has indeed thermalized to a Maxwellian by the end of the simulation $\left(t=5 \times 10^{-2} \approx 20 \tau\right)$. 

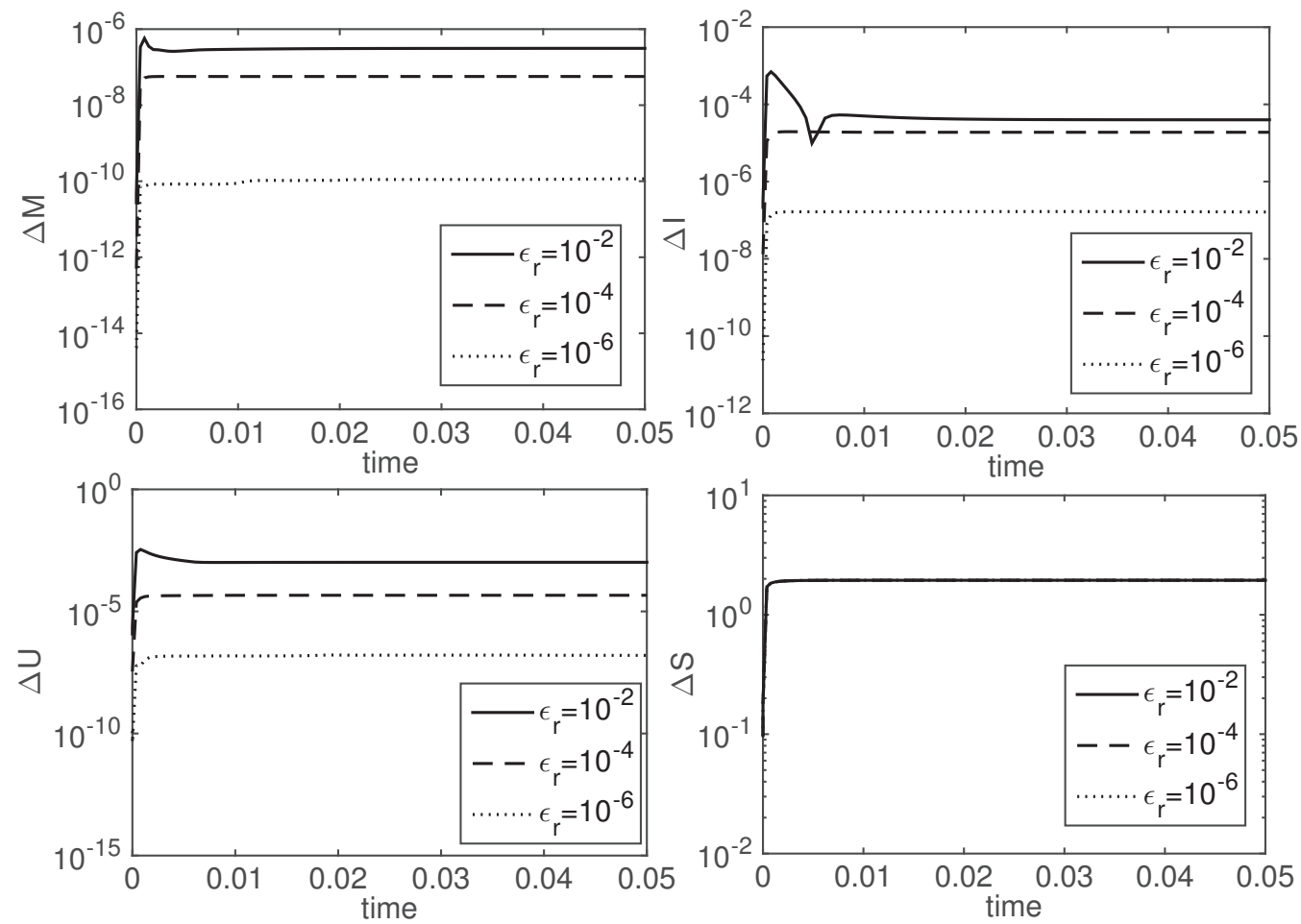

Figure 5.8: Single-species random distribution: Conservation properties and entropy change as a function of time.
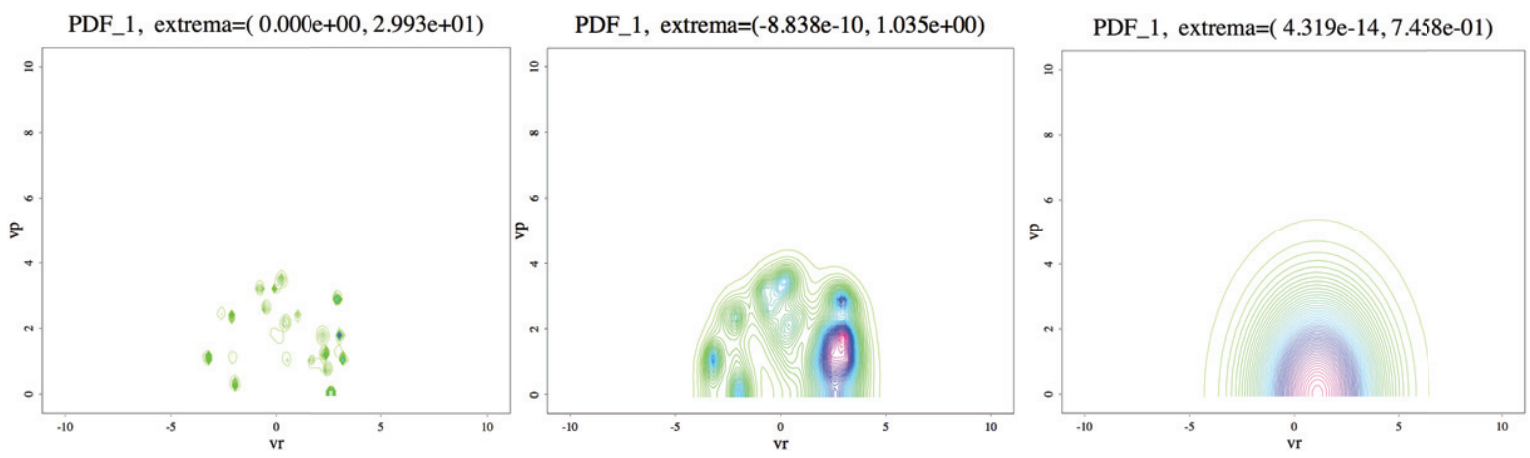

Figure 5.9: Single-species random distribution: The distribution function for the random initial distribution case at $\mathrm{t}=0$ (left), $t=2 \times 10^{-4}$ (middle), and $t=5 \times 10^{-2}$ (right) time. 

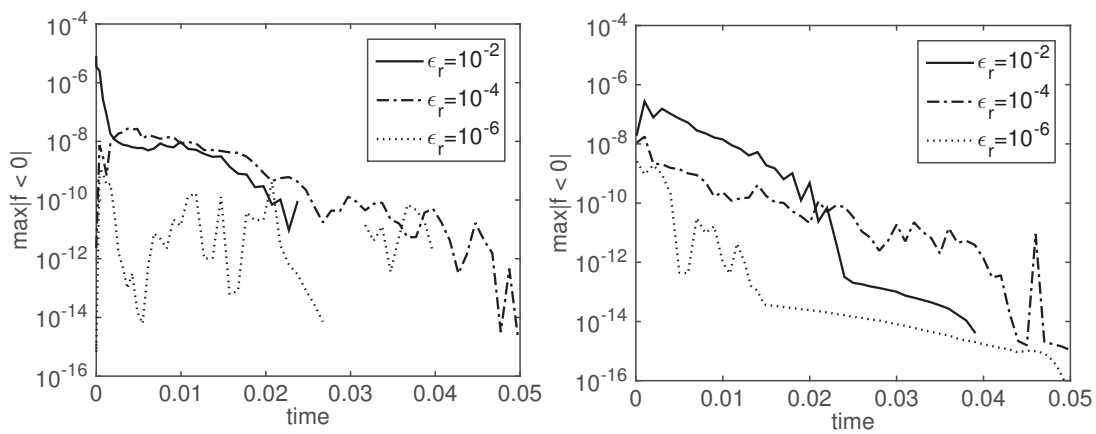

Figure 5.10: Single-species random distribution: The quality of positivity as a function of time for various values of nonlinear tolerance $\epsilon_{r}$ and time-stepping approach (BDF2, left, and BDF1, right). Temporal locations without values indicate strict positivity.

Regarding positivity, this is a more challenging test than the equilibrium solution. In fact, Fig. 5.9 shows that a negative value of order $\mathcal{O}\left(10^{-10}\right)$ is encountered at the intermediate time. To characterize the quality of positivity preservation, Fig. 5.10 depicts the time history of the magnitude of negative values of $f$ as a function of nonlinear tolerance $\epsilon_{r}$ and time-stepping approach (BDF1 and BDF2). It is seen that the quality of positivity is better for BDF1 than BDF2, as expected from Ref. [41], and that it correlates with $\epsilon_{r}$. Positivity also improves as $t \rightarrow \infty$ and the solution approaches a steady state.

\subsection{Two-species thermal equilibration}

We consider a two-species thermal equilibration case to compare the solution of the Fokker-Planck equation against a semi-analytical thermalization model. Two species with $m_{1}=2, m_{2}=4, Z_{1}=1, Z_{2}=2$, $n_{1}=n_{2}=1, u_{\|, 1}=u_{\|, 2}=0, T_{1}=1, T_{2}=25$, and a velocity space domain of $v_{\|} \in\left[-15 v_{t h, r e f}, 15 v_{t h, r e f}\right]$ and $v_{\perp} \in\left[0,15 v_{t h, r e f}\right]$ are considered. A large velocity domain is needed to accommodate the temperature disparity. Here, $v_{t h, r e f}=\sqrt{2 T_{r e f} / m_{\text {ref }}}=1$ is the reference thermal velocity with $T_{\text {ref }}=0.5$ and $m_{\text {ref }}=1$. In Fig. 5.11, temperatures are plotted as a function of time, and compared against a numerical solution to the following asymptotic temperature evolution ODE [7]:

$$
\frac{\partial T_{\alpha}}{\partial t}=\frac{\left(T_{\alpha}-T_{\beta}\right)}{\tau_{\alpha \beta}}
$$

where

$$
\tau_{\alpha \beta}^{-1}=\left(\frac{8}{\sqrt{\pi}} \frac{\sqrt{m_{\alpha} m_{\beta}}\left(Z_{\alpha} Z_{\beta}\right)^{2} \Lambda_{\alpha \beta}}{\left(m_{\alpha} T_{\beta}+m_{\beta} T_{\alpha}\right)^{3 / 2}}\right)
$$

is the interspecies thermal relaxation rate.

Good agreement is found in Fig. 5.11 between the fully kinetic numerical simulation and the semianalytical reference solution. In Fig. 5.12, the conservation properties and entropy change are shown as a function of time and nonlinear tolerance. Again, conservation is enforced down to the nonlinear convergence tolerance (except for parallel momentum, which is enforced to numerical round-off by symmetry), and entropy grows until the two species thermally equilibrate. We demonstrate second order time convergence of the BDF2 scheme by computing the $L_{2}$-norm of difference in solution against a reference solution,

$$
L_{2}^{\Delta t}=\sum_{\alpha=1}^{N_{s}} \sqrt{\left\langle f_{\alpha, j, k}^{\Delta t}-f_{\alpha, j, k}^{\Delta t_{r e f}}, f_{\alpha, j, k}^{\Delta t}-f_{\alpha, j, k}^{\Delta t_{r e f}}\right\rangle_{\vec{v}}}
$$

Here, $f^{\Delta t_{r e f}}$ is the solution obtained using a reference time-step size $\left(\Delta t_{r e f}=10^{-4}\right)$; refer to Figure 5.13. As can be seen, second-order convergence is realized with $\Delta t$ refinement.

We use this test case to study the performance of the multigrid preconditioned JFNK solver. This problem features multiple dynamical time-scales (self-collision time-scale and inter-species relaxation time-scale), and 


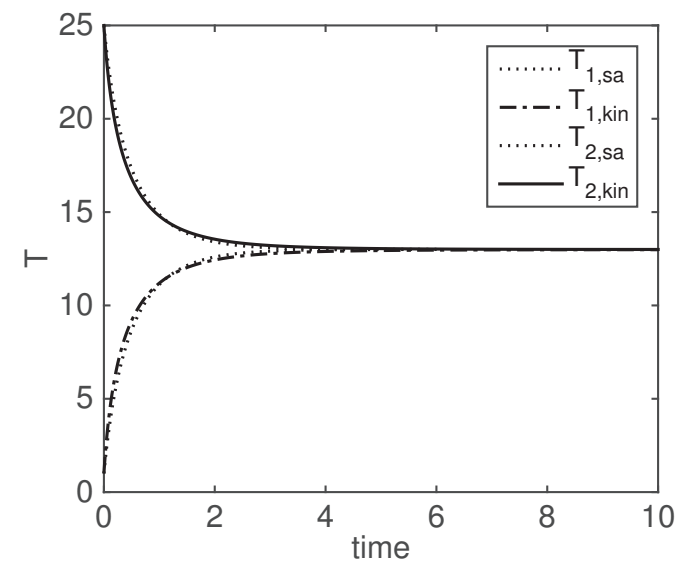

Figure 5.11: Two-species thermal equilibration: Temperature equilibration and comparison against a semi-analytical model.
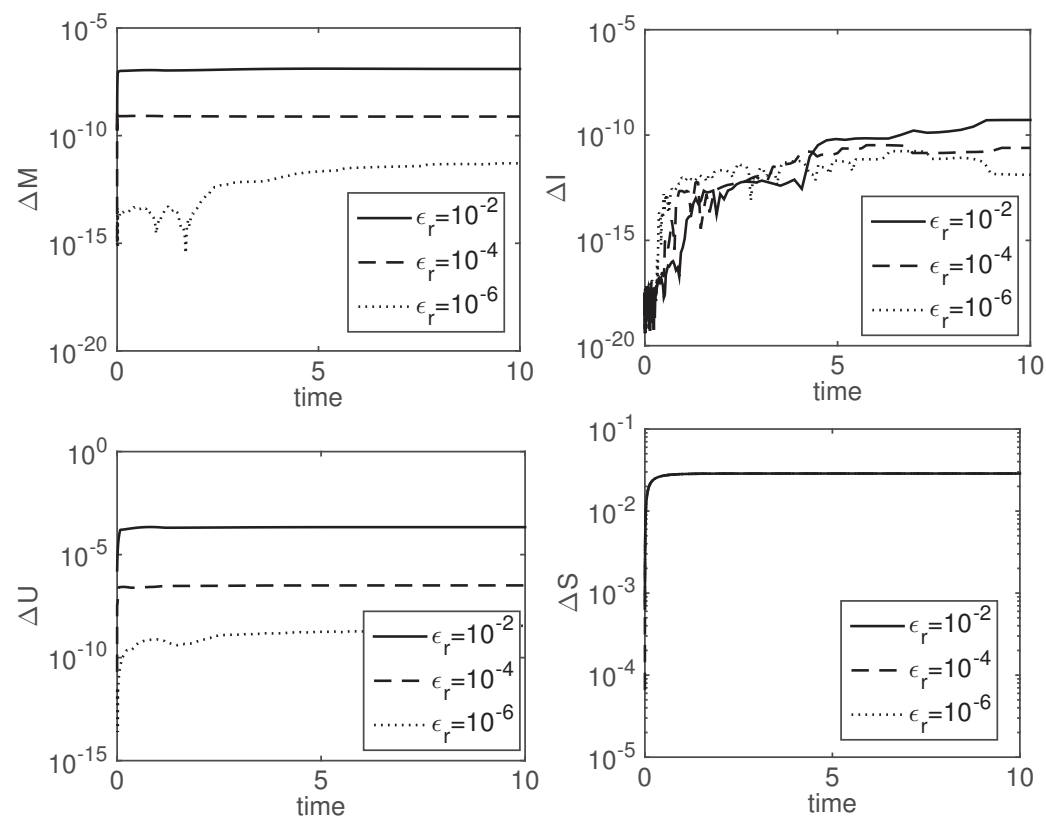

Figure 5.12: Two-species thermal equilibration: Conservation properties and entropy as a function of time and nonlinear tolerance. The apparently sharp evolution of some of these quantities early in time (in constrast with the relatively slow evolution of the temperature equilibration in the previous figure) is an artifact of the use of logarithmic units in the ordinates.

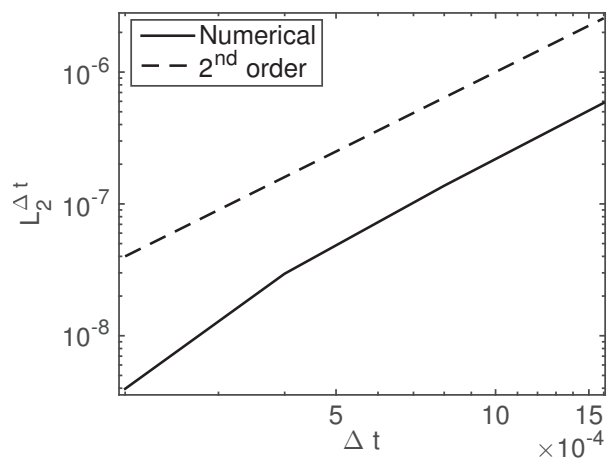

Figure 5.13: Two-species thermal equilibration: Demonstration of second-order convergence of the BDF2 scheme. 
Table 1: Two-species thermal equilibration: Performance of a preconditioned (left) and unpreconditioned (right) JFNK solver for different time-step and grid sizes for the thermal equilibration problem. The relative convergence tolerance was set to $\epsilon_{r}=10^{-4}$. We report the average number of Krylov iterations per time step (over 10 time steps). The asterisk denotes that only a single time-step was performed while the dash denotes that no time-step was performed due to large CPU time requirements. The dagger denotes that averaging was done only for 3 time-steps because the temperature has equilibrated between the two species by then.

\begin{tabular}{|c|c|c|c|}
\hline$N_{v}$ & (Preconditioned Krylov/ $\Delta t)_{a v g}$ & (Unpreconditioned Krylov/ $\Delta t)_{a v g}$ & $\Delta t / \Delta t_{\text {exp }}$ \\
\hline \multicolumn{4}{|c|}{$\Delta t=10^{-3}=2.4 \times 10^{-2} \tau_{\text {cold }}$} \\
\hline $128 \times 64$ & 2.7 & 3.9 & 6.25 \\
\hline $256 \times 128$ & 2.8 & 7.5 & 28.6 \\
\hline $512 \times 256$ & 2.7 & 12.6 & 122.0 \\
\hline \multicolumn{4}{|c|}{$\Delta t=10^{-2}=2.4 \times 10^{-1} \tau_{\text {cold }}$} \\
\hline $128 \times 64$ & 3.2 & 15.7 & 62.5 \\
\hline $256 \times 128$ & 3.2 & 38.6 & 286 \\
\hline $512 \times 256$ & 3.2 & 101.3 & 1220 \\
\hline \multicolumn{4}{|c|}{$\Delta t=10^{-1}=2.4 \tau_{\text {cold }}$} \\
\hline $128 \times 64$ & 4.9 & $72^{*}$ & 625 \\
\hline $256 \times 128$ & 4.3 & $228^{*}$ & 2860 \\
\hline $512 \times 256$ & 4.4 & $783^{*}$ & 12200 \\
\hline \multicolumn{4}{|c|}{$\Delta t=10^{0}=24 \tau_{\text {cold }}$} \\
\hline $128 \times 64$ & $8.7^{\dagger}$ & 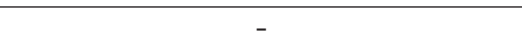 & 6250 \\
\hline $256 \times 128$ & $7.7^{\dagger}$ & - & 28600 \\
\hline $512 \times 256$ & $9.0^{\dagger}$ & - & 122000 \\
\hline
\end{tabular}

is therefore stiff. Table 1 contains JFNK performance data for a multigrid preconditioned solver and an unpreconditioned one, for various $\Delta t$. Here, $\Delta t_{\text {exp }}$ is the explicit time-step size computed as:

$$
\Delta t_{\text {exp }}=\min _{\alpha=1, \ldots, N_{s}}\left\{0.25\left(\frac{\Delta v_{\|}^{2}}{D_{\alpha,\|,\|}^{*}}\right), 0.25\left(\frac{\Delta v_{\|}^{2}}{D_{\alpha, \perp, \perp}^{*}}\right),\left(\frac{\Delta v_{\|}}{A_{\alpha, \|}^{*}}\right),\left(\frac{\Delta v_{\perp}}{A_{\alpha, \perp}^{*}}\right)\right\},
$$

where $D_{\alpha}^{*}$ and $A_{\alpha}^{*}$ are the effective transport coefficients, defined as:

$$
D_{\alpha}^{*}=\sum_{\beta=1}^{N_{s}} \Gamma_{\alpha \beta} D_{\beta}
$$

and

$$
A_{\alpha}^{*}=\sum_{\beta=1}^{N_{s}} \Gamma_{\alpha \beta} \frac{m_{\alpha}}{m_{\beta}} A_{\beta}
$$

The impact of the preconditioner in the performance of the method is evident, both in its ability to produce mesh-independent convergence rates, and as compared to the unpreconditioned simulations, resulting in a factor of $\sim 180$ improvement in the number of iterations for $\Delta t=10^{-1}$ with $N_{v}=512 \times 256$. It is also seen that, with preconditioning, the solver performance is quite insensitive to $\Delta t$ except for the case of $\Delta t=1$, which steps over the dynamical time-scale of the problem, $\tau_{\alpha \beta}=0.5$. Figure 5.14 shows the total CPU time of the second-order BDF2 implicit solver vs. a second-order Runge-Kutta explicit solver [54], as a function of the problem size $N_{v}$ for $\Delta t=10^{-1}$ and $t_{\max }=10 \Delta t$. Given the large cost of the explicit solver, the explicit CPU time in this figure is estimated by extrapolation from the cost of a single step as:

$$
\mathrm{CPU}_{\text {exp }, \text { tot }}=\frac{10 \Delta t}{\Delta t_{\text {exp }}} \times \mathrm{CPU}_{\text {exp }, \text { avg }}
$$

where $\mathrm{CPU}_{\text {exp,avg }}$ is the average (over 100 time steps) CPU time per time-step for the explicit solver. As can be seen, the multigrid-preconditioned fully implicit solver scales optimally as $\mathcal{O}\left(N_{v}\right)$, while the explicit solver CPU time scales as $\mathcal{O}\left(N_{v}^{2}\right)$, as expected. A speedup of four orders of magnitude of implicit vs. our 


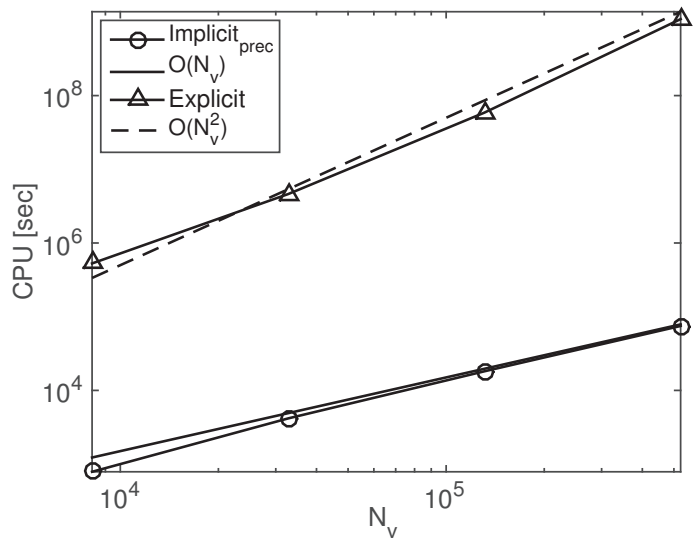

Figure 5.14: CPU time as a function of problem size $\left(N_{v}\right)$ for the preconditioned implicit $\left(\Delta t=10^{-1}\right)$ and explicit method.
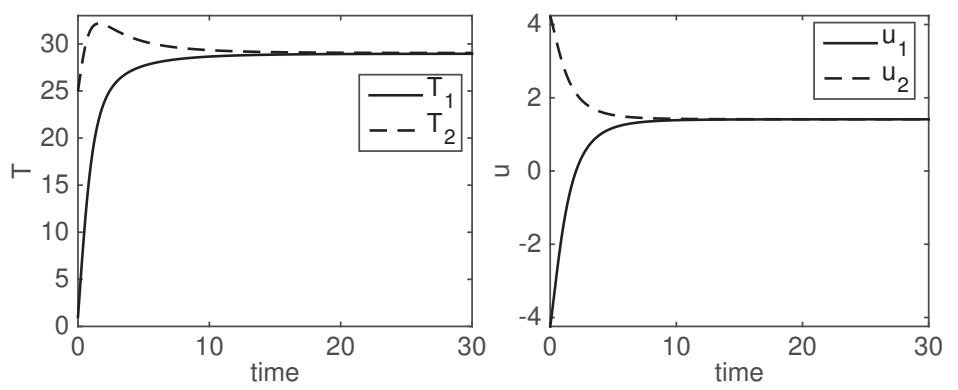

Figure 5.15: Two-species thermal and momentum equilibration: Temperature and momentum time history for both species.

simple explicit implementation is demonstrated for the finest mesh considered $(1024 \times 512)$. The coefficient of the scaling law for the explicit algorithm can be reduced by clever algorithmic strategies (see e.g. [42]), but the exponent cannot, and reflects a fundamental limitation of explicit approaches.

\subsection{Two Species Thermal and Momentum Equilibration}

We consider a final test case where two species with different initial velocity drifts and temperatures equilibrate in time. Specific parameters are $m_{1}=2, m_{2}=4, Z_{1}=1, Z_{2}=2, n_{1}=n_{2}=1, T_{1}=1$, $T_{2}=25$, and drifts $u_{\|, 1}=-3 v_{t h, \text { ref }}$ and $u_{\|, 2}=3 v_{t h, \text { ref }}$. The correct equilibrium values for temperature and momentum can be computed from total energy and momentum conservation:

$$
\begin{gathered}
\sum_{\alpha}\left(\frac{3}{2} n_{\alpha} T_{\alpha}+\frac{m_{\alpha} n_{\alpha} u_{\alpha}^{2}}{2}\right)^{0}=\sum_{\alpha}\left(\frac{3}{2} n_{\alpha} T_{\alpha}+\frac{m_{\alpha} n_{\alpha} u_{\alpha}^{2}}{2}\right)^{n} \\
\sum_{\alpha} I_{\alpha}=\sum_{\alpha}\left(m_{\alpha} n_{\alpha} u_{\|, \alpha}\right)^{0}=\sum_{\alpha}\left(m_{\alpha} n_{\alpha} u_{\|, \alpha}\right)^{n}
\end{gathered}
$$

where superscripts denote the time-level. The initial total momentum and energy are $I^{0}=8.49$ and $U^{0}=93$. From momentum conservation, $u_{1}^{\infty}=u_{2}^{\infty}=1.41$ and from energy conservation, $T_{1}^{\infty}=T_{2}^{\infty}=29$. In Fig. 5.15 , the temperature and momentum of the two species are shown as a function of time. As can be seen, the correct equilibrium values are achieved. In Fig. 5.16, we depict the conservation properties as well as entropy as a function of time. As before, conservation properties are enforced at or below the nonlinear tolerance level, and the physical trend in entropy is obtained.

\section{Conclusion}

In this study, we have developed and demonstrated for the first time an optimal, fully implicit algorithm for the multi-species, multidimensional Rosenbluth-Fokker-Planck equation. The algorithm features exact 

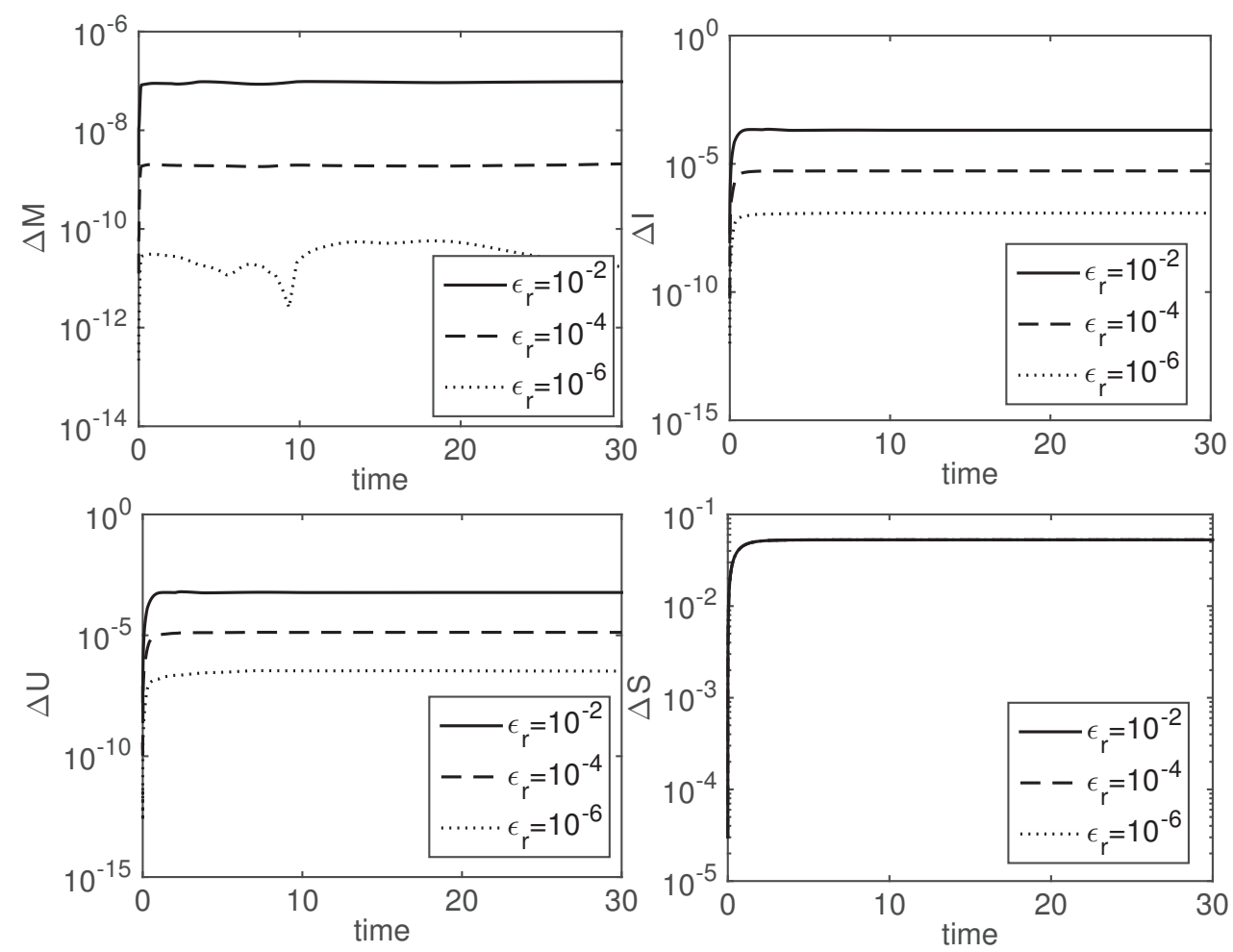

Figure 5.16: Two-species thermal and momentum equilibration: Conservation properties and entropy change in time as a function of nonlinear convergence tolerance.

(in practice, up to nonlinear tolerance) mass, momentum, and energy conservation, and excellent positivity preservation properties. Unlike earlier studies, our approach is based on the Rosenbluth form of the FokkerPlanck collision operator, which allows for an optimal implicit treatment using multigrid-preconditioned Jacobian-free Newton-Krylov methods. Key to our approach is the use of nonlinear constraints to enforce the continuum symmetries of the collision operator in the discrete. Positivity preservation is achieved by exploiting the advection-diffusion nature of the Fokker-Planck equation, by borrowing suitable limiters in both advection (SMART [37]) and tensor-diffusion [39] operators.

Performance-wise, the approach is demonstrated to scale algorithmically in an optimal manner, both in terms of grid-refinement (demonstrating grid-independent convergence rates and CPU scaling linearly with system size) and time-step size (showing very weak dependence). Four orders of magnitude speedup has been demonstrated vs. explicit approaches for the finest meshes considered $(1024 \times 512)$. The accuracy and performance of our approach has been demonstrated with a set of challenging numerical examples, employing both single and multiple species, and non-equilibrium configurations. We have verified our implementation by comparing against known relaxation rates, and by demonstrating relaxation towards the correct equilibrium solutions (which, owing to exact conservation, can be sustained forever without drift). We have also demonstrated numerically that the entropy obeys the H-theorem, i.e., either it grows or is conserved at equilibrium.

Future work will focus on extending the approach proposed here for the Fokker-Planck system to the Vlasov-Fokker-Planck equation, and on enforcing the preservation of exact invariants in that context.

\section{Acknowledgments}

The authors would like to thank D. A. Knoll for useful discussions and the referees for many constructive suggestions leading to the improvement of the paper. This work was sponsored by the Thermonuclear Burn Initiative of the Advanced Simulation and Computing Program at Los Alamos National Laboratory. This work was performed under the auspices of the National Nuclear Security Administration of the U.S. 
Department of Energy at Los Alamos National Laboratory, managed by LANS, LLC under contract DEAC52-06NA25396. 
[1] M. N. Rosenbluth, W. M. Macdonald, and D. L. Judd, "Fokker-Planck equation for an inverse-square force," Phys. Rev., vol. 107, no. 1, pp. 1-6, 1957.

[2] A. A. Arsen'ev and O. E. Buryac, "On the connection between a solution of the Boltzmann equation and a solution of the Landau-Fokker-Planck equation," USSR Comput. Maths math. Phys., vol. 17, pp. 241-246, 1991.

[3] L. Desvillettes, "On asymptotics of the Boltzmann equation when the collisions become grazing," Transport Theory and Statistical Physics, vol. 21, no. 3, pp. 259-276, 1992.

[4] P. Degond and B. Lucquin-Desreux, "The Fokker-Planck asymptotics of the Boltzmann collision operator in the Coulomb case," Math. Models Meth. Appl. Sci., vol. 2, no. 2, pp. 167-182, 1992.

[5] T. Goudon, "On Boltzmann equations and Fokker-Planck asymptotics: Influence of grazing collisions," Journal of Statistical Physics, vol. 89, no. 3/4, pp. 751-776, 1997.

[6] L. D. Landau, "The kinetic equation in the case of Coulomb interaction," Zh. Eksper. i Theoret. Fiz., vol. 7 , no. 2, 1937 .

[7] R. D. Hazeltine and J. D. Meiss, Plasma Confinement. Redwood City, CA: Addison-Wesly Publishing Company, 1991.

[8] J. S. Chang and G. Cooper, "A practical difference scheme for Fokker-Planck equations," J. Comput. Phys., vol. 6, pp. 1-16, 1970.

[9] A. B. Langdon, "Conservative differencing of the electron Fokker-Planck transport equation," in CECAM Report of Workshop on the Flux Limiter and Heat Flow Instabilities in Laser-Fusion Plasmas, Universite Paris Sud, France, 1981, p. 69.

[10] T. H. Kho, "Relaxation of a system of charged particles," Phys. Rev. A, vol. 32, no. 1, pp. 666-669, July 1985.

[11] E. Larsen, C. Levermore, and J. Sanderson, "Discretization methods for one-dimensional Fokker-Planck operators," Journal of Computational Physics, vol. 61, pp. 359-390, 1985.

[12] E. M. Epperlein, "Implicit and conservative difference scheme for the Fokker-Planck equation," J. Comput. Phys., vol. 112, pp. 291-297, 1994.

[13] E. S. Yoon and C. S. Chang, "A Fokker-Planck-Landau collision equation solver on two-dimensional velocity grid and its application to particle-in-cell simulation," Phys. Plasmas, vol. 21, p. 032503, 2014.

[14] _ - "Erratum:" A Fokker-Planck-Landau collision equation solver on two-dimensional velocity grid and its application to particle-in-cell simulation"," Phys. Plasmas, vol. 21, p. 039905, 2014.

[15] M. S. Pekker and V. N. Khudik, "Conservative finite-difference schemes for the Fokker-Planck equation," USSR Comput. Maths. math. Phys., vol. 24, no. 3, pp. 206-210, 1984.

[16] M. Lemou and L. Mieussens, "Implicit schemes for the Fokker-Planck-Landau equation," SIAM Journal of Scientific Computing, vol. 27, no. 3, pp. 809-830, 2005.

[17] C. Buet and S. Cordier, "Conservative and entropy decaying numerical scheme for the isotropic FokkerPlanck-Landau equation," Journal of Computational Physics, vol. 145, pp. 228-245, 1998.

[18] Y. A. Berezin, V. N. Khudick, and M. S. Pekker, "Conservative finite-difference schemes for the FokkerPlanck equation not violating the law of an increasing entropy," Journal of Computational Physics, vol. 69, pp. 163-174, 1987.

[19] C. Buet, S. Cordier, P. Degond, and M. Lemou, "Fast algorithms for numerical, conservative, and entropy approximations of the Fokker-Planck-Landau equation," Journal of Computational Physics, vol. 133, pp. 310-322, 1997. 
[20] P. Degond and B. Lucquin-Desreux, "An entropy scheme for the Fokker-Planck collision operator of plasma kinetic theory," Numerische Mathematik, vol. 68, pp. 239-262, 1994.

[21] M. Lemou, "Multipole expansions for the Fokker-Planck-Landau operator," Numerische Mathematik, vol. 78 , pp. 597-618, 1998.

[22] O. Larroche, "Kinetic simulation of a plasma collision experiment," Physics of Fluids B: Plasma Physics, vol. 5, pp. 2816-2840, 1993.

[23] — , "Kinetic simulation of fuel ion transport in ICF target implosions," European Physical Journal D, vol. 27 , pp. 131-146, 2003.

[24] M. Lemou and L. Mieussens, "Fast implicit schemes for the Fokker-Planck-Landau equation," Analyse Numèrique, vol. 338, pp. 809-814, 2004.

[25] M. Casanova, O. Larroche, and J. Matte, "Kinetic simulation of a collisional shock wave in a plasma," Physical Review Letters, vol. 67, no. 16, pp. 2143-2146, 1991.

[26] F. Filbet and L. Pareschi, "A numerical method for the accurate solution of the Fokker-Planck-Landau equation in the nonhomogeneous case," Journal of Computational Physics, vol. 179, pp. 1-26, 2002.

[27] F. Filbet and S. Jin, "A class of asymptotic-preserving schemes for kinetic equations and related problems with stiff sources," Journal of Computational Physics, vol. 229, pp. 7625-7648, 2010.

[28] S. Jin and B. Yan, "A class of asymptotic-preserving schemes for the Fokker-Planck-Landau equation," Journal of Computational Physics, vol. 230, pp. 6420-6437, 2011.

[29] K. Molvig, A. N. Simakov, and E. L. Vold, "Classical transport equations for burning gas-metal plasmas," Physics of Plasmas, vol. 21, p. 092709, 2014.

[30] S. I. Braginskii, "Transport processes in a plasma," in Reviews of Plasma Physics, M. A. Leontovich, Ed. New York: Consultants Bureau, 1965, vol. 1, pp. 205-311.

[31] S. Chapman and T. Cowling, The Mathematical Theory of Non-Uniform Gases. Cambridge, UK: Cambridge University Press, 1970.

[32] L. Chacón, D. C. Barnes, D. A. Knoll, and G. H. Miley, "An implicit energy-conservative 2D FokkerPlanck algorithm: II-Jacobian-free Newton-Krylov solver," J. Comput. Phys., vol. 157, pp. 654-682, 2000.

[33] L. G. A. Pataki, "Fast elliptic solvers in cylindrical coordinates and the Coulomb collision operator," Journal of Computational Physics, vol. 230, pp. 7840-7852, 2011.

[34] L. Chacón, D. C. Barnes, D. A. Knoll, and G. H. Miley, "An implicit energy-conservative 2D FokkerPlanck algorithm: I- Difference scheme," J. Comput. Phys., vol. 157, no. 2, pp. 618-653, 2000.

[35] W. L. Briggs, A Multigrid Tutorial. Philadelphia, PA: SIAM, 1987.

[36] C. Buet and S. Cordier, "Numerical Analysis of the Isotropic Fokker-Planck-Landau Equation," J. Comput. Phys., vol. 179, pp. 43-67, 2002.

[37] P. H. Gaskell and A. K. C. Lau, "Curvature-compensated convective transport: SMART, a new boundedness-preserving transport algorithm," International Journal for Numerical Methods in Fluids, vol. 8, pp. 617-641, 1988.

[38] L. Chacón, "A non-staggered, conservative, $\nabla \cdot \vec{B}$, finite-volume scheme for $3 \mathrm{~d}$ implicit extended magnetohydrodynamics in curvilinear geometries," Journal of Computational Physics, vol. 163, pp. 143-171, 2004.

[39] P. Sharma and G. W. Hammett, "Preserving monotonicity in anisotropic diffusion," J. Comput. Phys., vol. 227, no. 1, pp. 123-142, NOV 102007. 
[40] B. V. Leer, "Towards the ultimate conservative difference scheme III. upstream-centered finite-difference schemes for ideal compressible flow," Journal of Computational Physics, vol. 23, pp. 263-275, 1977.

[41] K. Duraisamy, J. D. Baeder, and J. G. Liu, "Concepts and application of time-limiters to high resolution schemes," Journal of Scientific Computing, vol. 19, pp. 139-162, 2003.

[42] O. Larroche, "An efficient explicit numerical scheme for diffusion-type equations with a highly inhomogeneous and highly anisotropic diffusion tensor," Journal of Computational Physics, vol. 223, pp. 436-450, 2007.

[43] W. Taitano and L. Chacón, "Charge-and-energy conserving moment-based accelerator for a multi-species Vlasov-Fokker-Planck-Ampère system, part I: Collisionless aspects," Journal of Computational Physics, vol. 284, pp. 718-736, 2015.

[44] G. D. Byrne and A. C. Hindmarsh, "A polyalgorithm for the numerical solution of ordinary differential equations," ACM Transactions on Mathematical Software, vol. 1, no. 1, pp. 71-96, 1975.

[45] D. A. Knoll and D. E. Keyes, "Jacobian-free Newton-Krylov methods: a survey of approaches and applications," Journal of Computational Physics, vol. 193, pp. 357-397, 2004.

[46] C. T. Kelley, Iterative Methods for Linear and Nonlinear Equations. Philadelphia: SIAM, 1995.

[47] Y. Saad, "A flexible inner-outer preconditioned GMRES algorithm," SIAM J. Sci. Comput., vol. 14, no. 2, pp. 461-469, 1992.

[48] R. S. Dembo, S. C. Eisenstat, and T. Steihaug, "Inexact Newton methods," SIAM Journal of Numerical Analysis, vol. 19, no. 2, pp. 400-408, 1982.

[49] G. Chen, L. Chacón, and D. C. Barnes, "An energy- and charge-conserving, implicit, electrostatic particle-in-cell algorithm," Journal of Computational Physics, vol. 230, pp. 7018-7036, 2011.

[50] W. T. Taitano, Development of a Jacobian-Free-Newton-Krylov Method with Kinetic Enslavement to Implicitly Solve Vlasov-Poisson System in Plasma Physics. Idaho: University of Idaho, 2010.

[51] H. Park, D. A. Knoll, R. M. Rauenzahn, C. K. Newman, J. D. Densmore, and A. B. Wollaber, "An efficient and time accurate, moment-based scale-bridging algorithm for thermal radiative transfer problems," SIAM Journal of Scientific Computing, vol. 35, no. 5, pp. S18-S41, 2013.

[52] J. A. Willert, H. Park, and W. T. Taitano, "Applying nonlinear diffusion acceleration to the neutron transport k-eigenvalue problem with anisotropic scattering," Nuclear Science and Engineering, Accepted, 2015.

[53] — "Using Anderson acceleration to accelerate the convergence of neutron transport calculations with anisotropic scattering," Nuclear Science and Engineering, Accepted, 2015.

[54] J. C. Butcher, "Coefficients for the study of Runge-Kutta integration processes," Journal of Australian Mathematical Society, vol. 3, no. 2, pp. 185-201, 1962.

[55] B. P. Leonard, "A stable and accurate convective modelling procedure based on quadratic upstream interpolation," Computer Methods in Applied Mechanics and Engineering, vol. 19, pp. 59-98, 1979.

[56] D. Kuzmin, M. J. Shashkov, and D. Svyatskiy, "A constrained finite element method satisfying the discrete maximum principle for anisotropic diffusion problems," J. Comput. Phys., vol. 228, no. 9, pp. 3448-3463, MAY 202009. 